\title{
Kidney-Specific WNK1 Amplifies NCC Responsiveness to Potassium Imbalance
}

Cary R. Boyd-Shiwarski ${ }^{1}$, Rebecca T. Beacham ${ }^{1}$, Shawn E. Griffiths ${ }^{1}$, Daniel J. Shiwarski ${ }^{2}$, Sophia A. Knoell ${ }^{1}$, Lubika J. Nkashama ${ }^{1}$, Kate Querry ${ }^{1}$, Allison L. Marciszyn ${ }^{1}$, Chou-Long Huang ${ }^{3}$, Sean D. Stocker ${ }^{1,4}$, Arohan R. Subramanya ${ }^{1,5,6^{*}}$

${ }^{1}$ Department of Medicine University of Pittsburgh School of Medicine

${ }^{2}$ Department of Biomedical Engineering, Carnegie Mellon University

${ }^{3}$ Department of Internal Medicine, University of Iowa Carver College of Medicine

${ }^{4}$ Center for Neuroscience, University of Pittsburgh School of Medicine

${ }^{5}$ Department of Cell Biology, University of Pittsburgh School of Medicine

${ }^{6}$ VA Pittsburgh Healthcare System

*Corresponding Author: ars129@pitt.edu

\begin{abstract}
The distal convoluted tubule (DCT) $\mathrm{NaCl}$ cotransporter $\mathrm{NCC}$ is activated by phosphorylation, a process that is potassium-regulated and dependent on With-No-Lysine (WNK) kinases. KSWNK1, a kidney-specific WNK1 isoform lacking the kinase domain, controls WNK signaling pathway localization in the DCT. Its role in NCC regulation, however, is unresolved: while early studies proposed that KS-WNK1 functions as an NCC inhibitor, recent work suggests that it activates NCC. To evaluate the role of KS-WNK1 on potassium-dependent NCC regulation, we studied KS-WNK1-KO mice across a wide range of plasma $\mathrm{K}+(2.0-9.0 \mathrm{mmol} / \mathrm{L})$, induced by dietary maneuvers and diuretic challenges. Potassium-deprived KS-WNK1-KO mice exhibited low WNK-dependent NCC phosphorylation compared to littermates, indicating that KS-WNK1 activates NCC during $\mathrm{K}+$ deficiency. In contrast, relative NCC phosphorylation was high in potassium-loaded KS-WNK1-KO mice, consistent with KS-WNK1-mediated NCC inhibition during hyperkalemia. An integrated analysis revealed that KS-WNK1 expands the dynamic range of NCC responsiveness to potassium, steepening the linear inverse relationship between NCC phosphorylation and plasma $[\mathrm{K}+]$. The effect of KS-WNK1 deletion was strongest in potassiumrestricted females, as they developed exaggerated hypokalemia and thiazide insensitivity due to low NCC activity. Taken together, these findings indicate that KS-WNK1 is a potassiumresponsive signaling amplifier that converts small changes in $[\mathrm{K}+]$ into large effects on $\mathrm{NCC}$ phosphorylation. This effect predominates in females during potassium deficiency, when high $\mathrm{NCC}$ activity is required to maintain salt reabsorption without exacerbating $\mathrm{K}+$ losses. These observations define the role of KS-WNK1 in NCC regulation, and identify a novel mechanism that contributes to sexual dimorphism in the mammalian nephron.
\end{abstract}




\section{Introduction}

The distal nephron senses and maintains plasma $\mathrm{K}+$ concentrations within the narrow window required for cellular function. During potassium deficiency, the DCT basolateral membrane becomes hyperpolarized, triggering a kinase cascade that activates the thiazide-sensitive $\mathrm{NaCl}$ cotransporter (NCC) via phosphorylation [1]. Hypokalemia-mediated NCC activation limits downstream sodium transport in the connecting tubule and collecting duct, diminishing distal voltage-dependent potassium secretion. This minimizes urinary potassium losses to conserve total body $\mathrm{K}+$ [2]. In contrast, hyperkalemia inhibits NCC, facilitating kaliuresis [3]. With-No-Lysine (WNK) kinases are essential regulators of NCC phosphorylation and potassium homeostasis [4]. During hypokalemia, the WNKs activate the downstream kinases SPAK and OSR1, which phosphorylate NCC directly [1]. Conversely, hyperkalemia is associated with NCC dephosphorylation $[3,5]$. The coordinated action of these signals generates an inverse relationship between NCC phosphorylation status and plasma $\mathrm{K}+$ that is linear within the physiologic range [6].

WNK4 is the dominant DCT-expressed WNK kinase [7]. Though the full-length kinase-active "Long" isoform of WNK1 (L-WNK1) is also expressed in the DCT, its abundance is low [8]. Instead, the most abundant WNK1 isoform in the DCT is a truncated gene product that lacks intrinsic kinase activity. This isoform is termed "Kidney-Specific WNK1" (KS-WNK1) due to its exclusive expression in the distal tubule [8]. KS-WNK1 controls WNK-SPAK/OSR1 pathway localization in the DCT. During hypokalemia, DCT-expressed WNK4, SPAK, and OSR1 condense into WNK bodies- signaling puncta whose appearance correlates with NCC activation $[9,10]$. Potassium-restricted KS-WNK1 knockout mice fail to form WNK bodies, suggesting that KS-WNK1 functions as a DCT-specific scaffold for WNK signaling.

Despite an essential role for KS-WNK1 in regulating the spatial organization of the WNKSPAK/OSR1 pathway, its physiologic role in NCC regulation remains unresolved. Early studies suggested that KS-WNK1 functions as an NCC inhibitor [11-14]. However, more recent work indicates that KS-WNK1 can activate $\mathrm{NCC}[15,16]$. Here, we propose a solution that reconciles these findings, which emerged when we studied effects of KS-WNK1 on NCC phosphorylation across the physiologic spectrum of blood potassium concentrations.

\section{Results}

KS-WNK1 amplifies the inverse relationship between NCC phosphorylation and blood [K+]. Recent work has implicated KS-WNK1 in the formation of WNK bodies during potassium deficiency, suggesting it is active during hypokalemic stress [9]. To more fully assess the role of KS-WNK1 in NCC phosphorylation across the physiologic range of plasma [K+], KS-WNK1-KO mice and wild-type littermates (WT) were administered diets with low, standard, or high potassium content for 10 days. Since mice with normal GFR efficiently excrete a potassium load [17, 18], we supplemented the high $\mathrm{K}+$ diet-fed mice with amiloride $(2 \mathrm{mg} / \mathrm{kg} / \mathrm{day})$ to induce frank 
hyperkalemia. We then assessed NCC abundance and phosphorylation status via immunoblotting (Figs 1A,B). Total NCC (tNCC) abundance exhibited a linear inverse relationship with blood [K+], and KS-WNK1 deletion had no significant effect on tNCC expression (Fig 1C) or on the slope of the inverse relationship (Fig 1F). Therefore, total NCC protein expression in the DCT is inversely regulated by blood $[\mathrm{K}+]$ in a manner that does not require $\mathrm{KS}-\mathrm{WNK} 1$.

Next, we assessed the effect of KS-WNK1 on phosphorylated NCC (pNCC) as a function of systemic potassium concentrations. WT littermates replicated the previously reported inverse relationship between blood $[\mathrm{K}+]$ and NCC phosphorylation at threonine-53, a marker of NCC activation [6]. In contrast, female and male KS-WNK1-KO mice exhibited lower pNCC abundance during potassium restriction and higher $\mathrm{pNCC}$ abundance during $\mathrm{K}+$ loading with amiloride (Fig 1A,B,D). In the latter case, the increased signal was obvious only after prolonged membrane exposure. Nevertheless, similar results were observed when a different NCC phosphoactivation site at serine-71 was probed (Fig S2) [19]. Thus, KS-WNK1 knockout mice exhibited a blunted inverse relationship between potassium and NCC phosphorylation (Fig 1D,G). Normalization of pNCC to tNCC reinforced this finding, as WT mice had the expected steep inverse slope, while KS-WNK1-KO mice had a flattened slope approaching zero (Fig 1E,H). Thus, KS-WNK1 augments NCC phosphorylation during hypokalemia and dampens NCC phosphorylation during hyperkalemia. These data suggest a biphasic relationship between KSWNK1 and NCC phosphorylation status, in which KS-WNK1 functions as an NCC activator during $\mathrm{K}+$ deficiency and an NCC inhibitor during $\mathrm{K}+$ excess (Fig 1H). Collectively, these data suggest that KS-WNK1 expands the dynamic range of NCC phosphorylation in response to changes in blood $[\mathrm{K}+]$, converting small changes in potassium into large effects on $\mathrm{NCC}$ phosphorylation status.

\section{Effect of KS-WNK1 on regulators of NCC phosphorylation during dietary $\mathrm{K}+$ restriction.}

During hypokalemia, the DCT senses a fall in blood potassium levels via the basolateral $\mathrm{K}+$ channel Kir4.1, triggering a signal that causes the WNK-SPAK/OSR1 pathway to condense into WNK bodies- KS-WNK1-dependent structures that have been proposed to mediate NCC activation [9, 10] (Fig 2A). Thus, we evaluated WNK-SPAK/OSR1 pathway expression in K+ restricted KS-WNK1 knockout mice. KS-WNK1 deletion had no effect on the total protein levels of WNK4, SPAK, or OSR1 in female (Fig 2B,D) or male (Fig 2C,D) mice fed a control diet. Consistent with prior reports, $\mathrm{K}+$ restriction upregulated the expression of WNK4 and SPAK in WT mice relative to control diets with standard potassium content (Fig 2B,C,D) [1, 20]. In contrast, male and female $\mathrm{K}+$ restricted KS-WNK1-KO mice exhibited decreased WNK4 and SPAK total protein abundance relative to $\mathrm{K}+$ restricted WT littermates (Fig 2D). This was associated with blunted SPAK and OSR1 phosphorylation compared to WT mice, indicating lower activity. The abundance of the basolateral K+ sensor Kir 4.1 (Fig 2A) was unaffected by KS-WNK1 both under control and low $\mathrm{K}+$ dietary conditions (Fig 2B,C,D). 
Next, we evaluated the effects of KS-WNK1 deletion on WNK body formation. Consistent with our prior report, which only evaluated WNK bodies in male mice [9], $\mathrm{K}+$ restriction in females was associated with the formation of DCT-expressed WNK bodies that stained positive for WNK4, WNK1, and SPAK (Fig 3A,B). These structures were largely absent in potassium-deficient KSWNK1-KO mice regardless of sex (Fig 3A,B,D). As noted previously, some of the KS-WNK1KO DCTs exhibited WNK-specific puncta during $\mathrm{K}+$ restriction, though these foci were mislocalized to the basal pole (Fig 3B,D) [9]. Morphometric analysis (Fig 3C) revealed that while both sexes exhibited the same number of WNK bodies per cell during $\mathrm{K}+$ restriction (Fig 3D), females exhibited larger foci that were positioned closer to the tubular lumen (Fig 3E,F). These findings indicate that female and male mice exhibit differences in WNK body morphology, suggesting sex-specific differences in WNK-SPAK/OSR1 pathway functionality.

In agreement with a recent study [10], DCT WNK bodies contained phosphorylated (active) SPAK and OSR1 (Fig 3A-C). Though the cytoplasmic punctate staining of phosphorylated SPAK and OSR1 was absent in KS-WNK1-KO mice, apical staining was still present (Fig 3A,B). Since SPAK/OSR1 and downstream NCC phosphorylation status were diminished in KS-WNK1 knockout mice (Figs 1 and 2), these findings suggest that WNK bodies are linked to enhanced WNK-SPAK/OSR1 pathway activity during hypokalemia.

Effect of KS-WNK1 on blood and urine electrolytes. Because NCC activity is high during hypokalemia and negligible during hyperkalemia [17], KS-WNK1 should have strong effects on NCC during states of potassium deprivation. Thus, we evaluated the physiologic effects of dietary potassium restriction on electrolyte handling in $\mathrm{KS}-\mathrm{WNK} 1-\mathrm{KO}$ mice. We initially compared pooled male and female cohorts of KS-WNK1-KO mice and WT littermates. On control diet, KSWNK1 deletion had no effect on blood or urine electrolytes, weight, dietary intake, or urine volume (Table S1). In contrast, potassium restriction in KS-WNK1-KO mice was associated with hypernatremia and decreased urine osmolality (Fig 4A,B). However, no other significant differences between wild-type and KS-WNK1 knockout mice were noted when males and females were analyzed together (Fig 4C,D, Table S1).

Females and males exhibit significant differences in serum $[\mathrm{K}+], \mathrm{NCC}$ activation, urine osmolality, DCT structure [21-26], and WNK body morphology (Fig 3), suggesting that the WNK signaling pathway exhibits sexual dimorphism. Consistent with this, we observed diverging trends when blood and urine data from $\mathrm{K}+$ restricted mice were disaggregated by sex. When placed on a $\mathrm{K}+$ deficient diet, KS-WNK1-KO females exhibited more pronounced hypokalemia, hypernatremia, and reduced urine osmolality compared to WT females (Fig 4A-C). Differences in urine $[\mathrm{K}+]$ were not observed, though urine potassium concentrations were at the low limit of detection due to avid $\mathrm{K}+$ conservation (Tables $1 \&$ 2). $\mathrm{K}+$-restricted female $\mathrm{KS}-\mathrm{WNK} 1-\mathrm{KO}$ mice also tended to have higher urine osmolality compared to KS-WNK1-KO male mice (Fig 4B); similar sex differences have been observed in other studies $[25,26]$. In contrast to females, male KS-WNK1-KO mice 
exhibited no difference in blood $[\mathrm{K}+]$ during potassium restriction (Fig 4C), despite strong effects of KS-WNK1 deletion on pNCC abundance (Fig 1A). Male KS-WNK1-KO mice did, however, have significantly increased blood $\left[\mathrm{Ca}^{2+}\right]$ (Fig 4D) relative to WT littermates, and trended towards higher blood $\left[\mathrm{Na}^{+}\right]$and lower urine osmolality without reaching statistical significance (Fig 4A,B).

Aside from effects on NCC, the relative hypokalemia observed in $\mathrm{K}+$ restricted $\mathrm{KS}-\mathrm{WNK} 1-\mathrm{KO}$ females could additionally be due to effects on other factors that mediate potassium secretion in the distal nephron, such as aldosterone, the epithelial sodium channel $(\mathrm{ENaC})$, and the renal outer medullary potassium channel, ROMK. As reported previously, potassium restriction was associated with increased expression of the uncleaved form of the $\mathrm{ENaC}$ gamma subunit (Fig S3A,B) and reduced ROMK protein abundance (Fig S3A,C) [17]. Potassium-restricted female KSWNK1-KO mice exhibited lower gamma ENaC abundance relative to WT mice (Fig S3B). Since the knockout females were hypokalemic relative to littermates (Fig 4), these data suggest that $\mathrm{ENaC}$ expression is physiologically downregulated in the absence of KS-WNK1 to mitigate urinary $\mathrm{K}+$ losses. ROMK expression was unchanged in $\mathrm{KS}-\mathrm{WNK} 1-\mathrm{KO}$ female mice relative to WT sex-matched littermates (Fig S3B,C). Moreover, aldosterone levels were markedly reduced to an equivalent degree in WT and KS-WNK1-KO mice (Fig S3D). Collectively, these findings suggest that the hypokalemia is due to reduced NCC activity rather than a primary alteration in $\mathrm{ENaC}, \mathrm{ROMK}$, or aldosterone.

Role of KS-WNK1 in blood pressure regulation, salt sensitivity, and thiazide responsiveness. Given the importance of NCC in blood pressure regulation [2], we performed telemetric blood pressure measurements in KS-WNK1-KO mice. These studies were focused on females, since they exhibited more pronounced hypokalemia and hypernatremia than males (Fig 4), and thus would be more likely to manifest detectable blood pressure differences. Despite these electrolyte derangements, ten days of potassium deprivation yielded no differences in mean arterial pressure (MAP) between WT and KS-WNK1-KO females throughout the course of the protocol (Fig 5A). Moreover, though both experimental groups developed a salt-sensitive increase in blood pressure, no differences between knockout mice and control littermates were observed during saline loading (Fig 5A). In contrast to the $\mathrm{K}+$ restricted condition, female mice administered a standard control diet for 10 days did not exhibit changes in blood pressure, either at baseline or after saline-loading, regardless of KS-WNK1 genotype (Fig 5A).

Though female $\mathrm{K}+$-restricted KS-WNK1-KO mice exhibited no differences in MAP compared to WT controls, the low pNCC abundance in KS-WNK1-KO mice suggested that NCC activity is reduced. To test for this, we challenged K+-restricted, saline-loaded female WT and KS-WNK1KO mice with an intraperitoneal injection of hydrochlorothiazide (HCTZ; 25mg/kg). Daytime MAP was recorded for $6 \mathrm{~h}$ windows one day before, during, and one day after HCTZ injection. WT mice responded to HCTZ injection, with a significant $4.5 \mathrm{mmHg}$ decrease in daytime MAP. In contrast, KS-WNK1-KO mice were insensitive to HCTZ, with only a $1.7 \mathrm{mmHg}$ decrease in 
daytime MAP that did not reach significance (Fig 5B). Thus, K+-restricted KS-WNK1-KO mice exhibit lower NCC activity compared to WT controls, a finding that is consistent with the low pNCC abundance in hypokalemic KS-WNK1 knockout mice observed in Fig 1D. Collectively these findings indicate that while female $\mathrm{K}+$ restricted $\mathrm{KS}-\mathrm{WNK} 1-\mathrm{KO}$ mice are normotensive, they maintain their blood pressure via mechanisms that compensate for impaired NCC activation during hypokalemia.

\section{Discussion}

Early studies of KS-WNK1 function suggested that it functions as an inhibitor of NCC-mediated sodium transport [11-14]. More recent reports, however, have provided compelling evidence that KS-WNK1 activates NCC $[15,16]$. Thus, the current literature is confounded by apparently conflicting evidence. We hypothesized that differences in experimental design might account for the discrepancies in study results, and that the regulatory effects of KS-WNK1 on NCC depend on the conditions under which it is studied. To test this, we subjected KS-WNK1 knockout mice and littermate controls to various maneuvers designed to manipulate plasma potassium concentrations and NCC phosphorylation status across a wide physiologic range. We found that in KS-WNK1$\mathrm{KO}$ mice, pNCC abundance is reduced relative to WT mice when it is activated during hypokalemia, and increased relative to WT mice when it is suppressed during hyperkalemia. By integrating these results as a function of blood potassium concentrations, we show that the steep linear inverse relationship between NCC phosphorylation and serum $[\mathrm{K}+]$ is blunted in mice lacking KS-WNK1. Thus, our data indicate that KS-WNK1 expands the dynamic range of NCC phosphorylation in response to changes in potassium (Fig 6A).

NCC plays an essential role in the kidney's ability to adjust salt reabsorption in response to potassium imbalance. In order to carry out this process, the DCT must first sense small fluctuations in interstitial potassium concentrations, and then amplify those tiny changes into a robust phosphorylation-dependent signal that appropriately adjusts NCC activity according to physiological need. While recent work indicates that the basolateral potassium channel Kir4.1 functions as the DCT potassium sensor [27], our findings demonstrate that KS-WNK1 is an essential part of the signal amplification mechanism. In the absence of KS-WNK1, the dynamic range of NCC phosphorylation is blunted. In other words, the signaling machinery in the DCT is unable to optimally increase or decrease NCC phosphorylation in response to a shift in extracellular $[\mathrm{K}+]$. Thus, KS-WNK1 boosts the effectiveness of intracellular signaling in the DCT, enabling impactful changes in NCC function during shifts in potassium balance.

Though KS-WNK1 augments NCC phosphorylation during hypokalemia and dampens its phosphorylation during hyperkalemia (Fig 6A), the physiological consequences of these actions are not equivalent. Hypokalemia is a potent stimulator of NCC activity. Thus, the blunted NCC phosphorylation observed in potassium-deprived KS-WNK1 knockout mice translates into significant changes in electrolyte handling and thiazide-responsiveness. By contrast, during 
extreme hyperkalemic conditions induced by the combination of dietary potassium loading and amiloride, pNCC abundance is essentially zero, and KS-WNK1 deletion results in only a slight increase in the cotransporter's phosphorylation status. Indeed, the KS-WNK1-dependent change in pNCC abundance by immunoblotting was so small that it was obvious only after prolonged membrane exposure (Fig 1B). Moreover, thiazide-sensitive $\mathrm{NaCl}$ cotransport during extreme hyperkalemic states is essentially undetectable: under such conditions, distal ENaC-mediated sodium transport predominates, to couple sodium reabsorption to voltage-dependent $\mathrm{K}+$ secretion [17]. Taken together, these findings strongly suggest that the functional effect of KS-WNK1mediated suppression of NCC activity during hyperkalemia is negligible, and that the primary physiologic role of KS-WNK1 in the DCT is to amplify NCC activity during hypokalemia. This fits well with the role of KS-WNK1 in the formation of hypokalemic WNK bodies, structures that are present in the DCT when NCC is highly active [9] and require KS-WNK1 for proper assembly (Fig 6B).

Recently, KS-WNK1 was linked to human disease. In 2020, Louis-Dit-Picard et al reported that a missense mutation of WNK1 that preferentially increases KS-WNK1 protein expression in the DCT causes Spitzer-Weinstein Syndrome, an inherited thiazide-curable form of hyperkalemic (Type IV) renal tubular acidosis with normal blood pressure $[15,28]$. Mice harboring this mutation developed normotensive thiazide-sensitive hyperkalemia, increased phosphorylation of SPAK and NCC on standard diet, and enlarged WNK bodies [15]. Our results indicate that KS-WNK1-KO mice are a mirror image of this gain-of-function mutant, as the $\mathrm{K}+$ restricted knockout mice lacked WNK bodies, and exhibited exaggerated hypokalemia, thiazide insensitivity, and reduced SPAK and NCC phosphorylation. The linkage of KS-WNK1 overexpression to a syndrome of NCC hyperactivation and thiazide-sensitive hyperkalemia further supports the physiological importance of KS-WNK1 as a DCT-specific signal amplifier for salt transport.

Thiazide diuretics commonly cause hypokalemia by increasing distal potassium secretion and hypercalcemia via enhanced proximal tubule calcium reabsorption [29, 30]. We propose that the changes to $[\mathrm{K}+]$ and $\left[\mathrm{Ca}^{2+}\right]$ in $\mathrm{KS}-\mathrm{WNK} 1-\mathrm{KO}$ mice are due to the combined effects of low NCC activity phenocopying the effects of thiazides, and sex-specific differences in electrolyte handling along the renal tubule. $\mathrm{K}+$ restricted female $\mathrm{KS}-\mathrm{WNK} 1-\mathrm{KO}$ mice exhibited hypokalemia with normocalcemia while male knockouts developed hypercalcemia with normokalemia. Females have expanded DCTs, increased NCC abundance, and a greater tendency to develop thiazideinduced hypokalemia [21-23, 31-33]. Males on the other hand tend to have increased proximal tubule mass, and thus are dependent on proximal rather than distal calcium reabsorption [23, 34, 35]. Thiazide-induced hypocalciuria is thought to be due to increased proximal tubule $\mathrm{Na}+$ and $\mathrm{Ca}+$ reabsorption caused by low NCC activity $[30,36]$. Thus, our results suggest that K-restricted male KS-WNK1-KO mice compensate for the reduction in NCC activity by upregulating proximal tubule sodium and calcium transport, leading to hypercalcemia. In contrast, female KS-WNK1$\mathrm{KO}$ mice are more dependent on the distal tubule for electrolyte handling, and have a phenotype 
that is predominantly reflected by distal $\mathrm{K}+$ wasting, leading to overt hypokalemia. These observations implicate KS-WNK1 as an important determinant of distal tubule sexual dimorphism, and suggest that females require WNK bodies to maintain potassium homeostasis.

The mechanism by which KS-WNK1 amplifies WNK-dependent signaling remains an open question. KS-WNK1 is devoid of intrinsic kinase activity due to the absence of a functional kinase domain. Its unique $\mathrm{N}$-terminus facilitates the formation of $\mathrm{WNK}$ bodies, while the $\mathrm{C}$-terminus binds WNK-SPAK pathway components and restricts them to specific cytosolic locations depending on systemic $\mathrm{K}+$ concentrations [9]. This suggests that KS-WNK1 functions as a DCTspecific scaffold for the WNK-SPAK/OSR1 pathway. We propose that the ability of KS-WNK1 to drive WNK body formation is linked to its function as a signal amplifier. During hypokalemia, KS-WNK1 likely organizes the WNK-SPAK/OSR1 pathway in such a manner that allows it to be efficiently activated. Thus, similar to other membraneless cytoplasmic foci [37], a major function of KS-WNK1-dependent WNK bodies may be to condense the WNK pathway within "reaction crucibles" that coordinate distal tubule electrolyte transport. Though the molecular basis by which WNK bodies amplify the WNK-SPAK/OSR1 pathway remains unresolved, understanding the mechanisms involved will provide insight into DCT function, and perhaps more broadly, how specific multicomponent signaling networks can be tuned for physiological benefit.

In conclusion, our findings provide insight into the role of KS-WNK1 in potassium-dependent NCC regulation. They implicate KS-WNK1 as a DCT-specific WNK signaling amplifier that optimizes NCC reactivity to changes in systemic potassium concentrations. This observation reconciles conflicting data regarding stimulatory vs inhibitory effects of KS-WNK1 on NCC. In addition, these results identify KS-WNK1 as a kidney-specific factor that contributes significantly to the sexual dimorphism of the renal tubule.

\section{Methods}

Animal studies. All animal protocols conform to the National Institutes of Health (NIH) Guide for the Care and Use of Laboratory Animals and were approved by the University of Pittsburgh IACUC. KS-WNK1 knockout mice (KS-WNK1-KO) and age-matched wild-type littermates (WT) were generated in a 129/Sv background as previously described [9, 13]. Experiments were performed on both female (20-30g) and male mice (25-35g), aged 10-25 weeks.

All mice were housed in a temperature-controlled room on a $12 \mathrm{~h}$ light/dark cycle. Mice had free access to deionized water, unless otherwise noted. KS-WNK1-KO mice were derived from a mouse line originally reported by Liu et al. [13] and described in the supplemental materials from Boyd-Shiwarski et al. [9]. Genotyping was performed as reported by these studies previously. The expected sizes of the WT and KS-WNK1-KO genotyping PCR products $(0.74 \mathrm{~kb}$ and $0.4 \mathrm{~kb}$, respectively). 
To determine the effect of KS-WNK1 on NCC phosphorylation, mice were fed varying $\mathrm{K}+$ diets for 10 days (low $\mathrm{K}+(<0.003 \% \mathrm{TD} .88239)$, control $\mathrm{K}+(1 \% \mathrm{TD} .88238)$, or high $\mathrm{K}+(5 \% \mathrm{~K}$ with 1:1:1 ratio of $\mathrm{K}+$ citrate, $\mathrm{KCl}$, and $\mathrm{K}+$ carbonate TD.07278) (Teklad, Madison, WI). To induce hyperkalemia, mice fed the high $\mathrm{K}+$ diet were supplemented with amiloride $(2 \mathrm{mg} / \mathrm{kg} / \mathrm{d})$ in their drinking water. The $\mathrm{Na}+$ content of all diets was $0.3 \%$. After 10 days, mice were anesthetized, and blood was obtained via terminal cardiac puncture and analyzed by iSTAT (Abbot). Kidneys were harvested and flash frozen for immunoblot and/or paraformaldehyde-treated for microscopy. Urine was immediately collected from mouse bladder for urine $\mathrm{pH}$ measurements using AimStrip US-5 (Germaine Inc, San Antonio, TX). Blood plasma was isolated by centrifugation and aldosterone levels were measured using ELISA kit (ENZO, ADI-900-173).

Metabolic Cages. To determine the effect of KS-WNK1 on blood and urine parameters, mice were individually housed in metabolic cages (Tecniplast, Italy). Mice were fed pellet-based diets during days 1-7, introduced to gel diets on day 7, and then switched to exclusive gel-diets for days 8-10. Gel diets were used to prevent pellet debris in the urine while in the metabolic cages. Gels diets were derived from blended commercial diets combined with 1-2\% agar (Fisher Scientific). The mice were acclimated in metabolic cages for the first $24 \mathrm{~h}$, followed by $24 \mathrm{~h}$ measurement of food and water intake and urine collection. Urine $[\mathrm{Na}+]$ and $[\mathrm{K}+]$ were determined using Easy Lyte Plus $\mathrm{Na} / \mathrm{K} / \mathrm{Cl}$ analyzer (Medica Corp, Bedford, MA). Urine osmolality was determined using a micro osmometer (Precision Systems, Natick, MA). After 10 days mice were anesthetized, blood obtained via terminal cardiac puncture and analyzed by iSTAT (Abbot). Kidneys were processed for immunoblot and imaging as stated below.

Immunoblotting. For protein quantification, kidney cortexes were flash frozen and processed as previously described [17]. Ice-cold RIPA buffer (Thermo Scientific) was used for protein extraction with freshly added protease and phosphatase inhibitor cocktail. Protein quantification was determined using the Pierce BCA Protein Assay Kit (Thermo Scientific). Uniform protein loading was determined using Coomassie-stained gels, as previously described $[17,38]$. 15 $\mu \mathrm{g}$ protein from each sample was loaded onto the SDS-PAGE gel and then stained with Coomassie blue. Five random bands were quantified and appropriate adjustments were made to the sample preparation until the loading was determined to be uniform. Final optimized gels are shown in Fig S1. Next, equal amounts (20-40ug) of protein were separated by SDS-PAGE using 4-20\% Criterion TGX precast gels (Bio-Rad). Protein was transferred to a nitrocellulose membrane. Signal densitometry was measured using Bio-Rad ChemiDoc and densitometry was quantified with ImageLab analysis software (Bio-Rad). Two different protein ladders were used: Precision Plus All Blue (Biorad) and PageRuler Plus (Thermo Scientific).

Antibodies. The following antibodies were used for immunoblot and/or immunofluorescence: Sodium chloride cotransporter (NCC; kindly provided by David Ellison [39]); Sodium chloride cotransporter (NCC; Millipore Ab3553 [40]); Phospho-NCC Thr 53 (pNCC; Phospho-solutions 
P1311-53 [41]); Phospho-NCC Ser 71 (pNCCSer71, kindly provided by Jan Loffing [3]); SPAK (SPAK; Cell Signaling [40]); Phospho-SPAK/ Phospho-OSR1 (pSPAK/pOSR1; Millipore 072273 [10]); With-no-lysine kinase 1 (WNK1; Atlas Antibodies HPA059157 [9]); With-no-lysine kinase 4, (WNK4 [9]); Gamma subunit epithelial sodium channel ( $\gamma \mathrm{ENaC} ; 83 \mathrm{kDa}$ cleaved and 95 kDa uncleaved; Stressmarq SPC-405)[17]; ATP-sensitive inward rectifier potassium channel 10 (Kir4.1; Alomone APC-035 [27]); Renal outer medullary potassium channel (ROMK; 50-65 kDa complex glycosylated; R-80 was kindly provided by James Wade)[42].

Blood Pressure Telemetry. Female KS-WNK1-KO mice and WT littermates were anesthetized with isoflurane and DSI PA-C10 telemetry units (Data Sciences International, New Brighton, MN, USA) were surgically implanted into the femoral artery as previously described [43]. Mice then recovered for 1 week before dietary challenges were commenced. Blood pressure was collected every day from 10am-4pm (daytime) and 10pm-4am (nighttime) for the duration of the diet challenge using Spike2 software (Cambridge Electronic Design). Mean arterial pressure (MAP) was calculated by diastolic plus one-third of the pulse pressure. For the saline challenge, mice were maintained on varying $\mathrm{K}+$ diets for 10 days and were then challenged with $1 \%$ saline in their drinking water for $72 \mathrm{~h}$. HCTZ-challenge was performed $72 \mathrm{~h}$ after saline administration commenced. During the HCTZ treatment mice were maintained on low $\mathrm{K}+$ diet with $1 \%$ saline drinking water. Daytime blood pressure was obtained for $6 \mathrm{~h}$ on 3 separate days: 1) the day prior to HCTZ administration, 2) the day of HCTZ administration, and 3) the day after HCTZ administration. Mice were injected with hydrochlorothiazide $(25 \mathrm{mg} / \mathrm{kg}$ IP) at $9 \mathrm{am}$ and then blood pressure was collected from 10am-4pm (daytime).

Quantitative Immunofluorescence Confocal Microscopy. Paraformaldehyde fixed kidney tissues were processed and prepared as previously described [17]. $6 \mu \mathrm{m}$ sections were rehydrated and treated with $1 \%$ SDS for 10 min for retrieval of antigenic sites. Then slides were washed with high salt buffer + bovine serum albumin before the addition of primary antibody. Primary antibodies were incubated overnight at $4^{\circ} \mathrm{C}$, followed by washes with high salt buffer + bovine serum albumin and subsequent incubation with secondary antibodies and TO-PRO-3 Iodide for visualize staining. Imaging of the kidney tissue was performed using a Leica HCX PL APO CS x40, 1.25 numerical aperture oil objective on a Leica TCS SP5 CW-STED confocal microscope utilizing Leica LasX software.

To produce quantitative measures for pSPAK puncta number, size, and distance to the DCT lumen we used Imaris (Bitplane, v9.5) image analysis software. Fluorescence images were first imported into Imaris. The pSPAK puncta were detected using the Spots element creation wizard. A region of interest was specified to isolate a single DCT within an image. The pSPAK channel was then selected as the fluorescence reference channel for both Spot identification and to guide the puncta diameter determination. A local contrast fluorescence intensity threshold was chosen, and the Spots elements were filtered using the Quality metric intrinsic to Imaris. The region growing 
method was used to obtain Spots of varying size. Once defined, the same quality metric and threshold settings were used throughout all puncta analysis. In an attempt to not exclude data, the quality and thresholding was set to bias towards over detection. Manual editing of the Spot objects was performed to eliminate rare errant Spots when necessary. For each DCT the number of cell nuclei were counted and used to calculate the average number of pSPAK puncta per cell. To measure the distance between the identified spots and the DCT lumen, we created a Surface object of the lumen. Using the Surface wizard, a Surface object was defined using the magic wand and isolines functions within the manual surface creation. This was able to reliably detect the lack of fluorescence intensity within the DCT lumen. A built-in Imaris Xtension was implemented to calculate the shortest distance between all Spots objects and the DCT lumen Surface. These data were then displayed in Imaris as Spot objects with color coded statistic values for puncta diameter or distance to DCT lumen.

Data analysis. Data were analyzed using Graph Pad Prism software and presented as mean \pm SEM, plus individual data points. Comparisons between two groups were determined by a Student's $t$ test. Comparisons between multiple groups were determined using one- or two-way analysis of variance (ANOVA), followed by the appropriate post hoc test. $\mathrm{P}$ values $\leq 0.05$ were considered statistically significant.

\section{Author Contributions}

C.B-S. and A.R.S. designed the study; C.B-S, R.T.B., S.A.K., S.E.G., L.J.N., K.Q., A.L.M., and S.D.S. performed experiments; C.B-S., D.J.S., S.D.S., S.A.K., and A.R.S. analyzed data; C.B-S., D.J.S., and A.R.S. made figures; C.B-S., R.T.B., S.E.G., D.J.S., S.D.S., and A.R.S. drafted the paper; C-L.H. provided KS-WNK1 knockout mice; all authors approved the final version of the manuscript.

\section{Acknowledgments}

This work was supported by National Institutes of Health grants K08DK118211 (to C.B-S.), R01DK098145 (to A.R.S.), R01DK1 19252 (to A.R.S.), R01DK111542 (to C-L.H.), P30DK79307 (Pittsburgh Center for Kidney Research). We thank Jan Loffing, David Ellison, and James Wade for antibodies and Tom Kleyman for helpful discussions. This content is solely the responsibility of the authors and does not necessarily represent the official views of the U.S. Department of Veterans Affairs. 


\section{References}

1. Terker, A.S., et al., Potassium modulates electrolyte balance and blood pressure through effects on distal cell voltage and chloride. Cell Metab, 2015. 21(1): p. 39-50.

2. Hoorn, E.J., et al., Regulation of the Renal NaCl Cotransporter and Its Role in Potassium Homeostasis. Physiol Rev, 2020. 100(1): p. 321-356.

3. Sorensen, M.V., et al., Rapid dephosphorylation of the renal sodium chloride cotransporter in response to oral potassium intake in mice. Kidney Int, 2013. 83(5): p. 811-24.

4. Wilson, F.H., et al., Human hypertension caused by mutations in WNK kinases. Science, 2001. 293(5532): p. 1107-12.

5. Picard, N., et al., Protein phosphatase 1 inhibitor-1 deficiency reduces phosphorylation of renal $\mathrm{NaCl}$ cotransporter and causes arterial hypotension. J Am Soc Nephrol, 2014. 25(3): p. 511-22.

6. Terker, A.S., et al., Unique chloride-sensing properties of WNK4 permit the distal nephron to modulate potassium homeostasis. Kidney Int, 2016. 89(1): p. 127-34.

7. Murillo-de-Ozores, A.R., et al., WNK4 Kinase: from structure to physiology. Am J Physiol Renal Physiol, 2021.

8. Vidal-Petiot, E., et al., A new methodology for quantification of alternatively spliced exons reveals a highly tissue-specific expression pattern of WNK1 isoforms. PLoS One, 2012. 7(5): p. e37751.

9. Boyd-Shiwarski, C.R., et al., Potassium-regulated distal tubule WNK bodies are kidneyspecific WNK1 dependent. Mol Biol Cell, 2018. 29(4): p. 499-509.

10. Thomson, M.N., et al., WNK bodies cluster WNK4 and SPAK/OSR1 to promote NCC activation in hypokalemia. Am J Physiol Renal Physiol, 2020. 318(1): p. F216-F228.

11. Yang, C.L., X.M. Zhu, and D.H. Ellison, The thiazide-sensitive Na-Cl cotransporter is regulated by a WNK kinase signaling complex. Journal of Clinical Investigation, 2007. 117(11): p. 3403-3411.

12. Subramanya, A.R., et al., Dominant-negative regulation of WNK1 by its kidney-specific kinase-defective isoform. Am J Physiol Renal Physiol, 2006. 290(3): p. F619-24.

13. Liu, Z., et al., Downregulation of NCC and NKCC2 cotransporters by kidney-specific WNK1 revealed by gene disruption and transgenic mouse models. Hum Mol Genet, 2011. 20(5): p. 855-66.

14. Hadchouel, J., et al., Decreased ENaC expression compensates the increased NCC activity following inactivation of the kidney-specific isoform of WNK1 and prevents hypertension. Proc Natl Acad Sci U S A, 2010. 107(42): p. 18109-14.

15. Louis-Dit-Picard, H., et al., Mutation affecting the conserved acidic WNK1 motif causes inherited hyperkalemic hyperchloremic acidosis. J Clin Invest, 2020. 130(12): p. 63796394.

16. Argaiz, E.R., et al., Kidney-specific WNK1 isoform (KS-WNK1) is a potent activator of WNK4 and NCC. Am J Physiol Renal Physiol, 2018. 315(3): p. F734-F745.

17. Boyd-Shiwarski, C.R., et al., Effects of extreme potassium stress on blood pressure and renal tubular sodium transport. Am J Physiol Renal Physiol, 2020. 318(6): p. F1341F1356.

18. Boyd-Shiwarski, C.R. and A.R. Subramanya, The renal response to potassium stress: integrating past with present. Curr Opin Nephrol Hypertens, 2017. 26(5): p. 411-418. 
19. Richardson, C., et al., Activation of the thiazide-sensitive Na+-Cl-cotransporter by the WNK-regulated kinases SPAK and OSR1. J Cell Sci, 2008. 121(Pt 5): p. 675-84.

20. Castaneda-Bueno, M., et al., Modulation of NCC activity by low and high $K(+)$ intake: insights into the signaling pathways involved. Am J Physiol Renal Physiol, 2014. 306(12): p. F1507-19.

21. Tahaei, E., et al., Distal convoluted tubule sexual dimorphism revealed by advanced 3D imaging. Am J Physiol Renal Physiol, 2020. 319(5): p. F754-F764.

22. Wysowski, D.K., et al., Sex and age differences in serum potassium in the United States. Clin Chem, 2003. 49(1): p. 190-2.

23. Veiras, L.C., et al., Sexual Dimorphic Pattern of Renal Transporters and Electrolyte Homeostasis. J Am Soc Nephrol, 2017. 28(12): p. 3504-3517.

24. Zheng, W., et al., Estrogens contribute to a sex difference in plasma potassium concentration: a mechanism for regulation of adrenal angiotensin receptors. Gend Med, 2006. 3(1): p. 43-53.

25. Frokiaer, J., et al., Low aquaporin-2 levels in polyuric DI $+/+$ severe mice with constitutively high cAMP-phosphodiesterase activity. Am J Physiol, 1999. 276(2): p. F17990.

26. Nair, A.V., et al., Sex-dependent differences in water homeostasis in wild-type and $V$ ATPase B1-subunit deficient mice. PLoS One, 2019. 14(8): p. e0219940.

27. Cuevas, C.A., et al., Potassium Sensing by Renal Distal Tubules Requires Kir4.1. J Am Soc Nephrol, 2017.

28. Margolis, B.L. and M.D. Lifschitz, The Spitzer-Weinstein syndrome: one form of type IV renal tubular acidosis and its response to hydrochlorothiazide. Am J Kidney Dis, 1986. 7(3): p. 241-4.

29. Subramanya, A.R. and D.H. Ellison, Distal convoluted tubule. Clin J Am Soc Nephrol, 2014. 9(12): p. 2147-63.

30. Nijenhuis, T., et al., Enhanced passive Ca2+ reabsorption and reduced $\mathrm{Mg} 2+$ channel abundance explains thiazide-induced hypocalciuria and hypomagnesemia. J Clin Invest, 2005. 115(6): p. 1651-8.

31. Toner, J.M. and L.E. Ramsay, Thiazide-induced hypokalaemia; prevalence higher in women. Br J Clin Pharmacol, 1984. 18(3): p. 449-52.

32. Verlander, J.W., et al., Estradiol enhances thiazide-sensitive NaCl cotransporter density in the apical plasma membrane of the distal convoluted tubule in ovariectomized rats. $\mathrm{J}$ Clin Invest, 1998. 101(8): p. 1661-9.

33. Clark, B.G., et al., Female preponderance in diuretic-associated hypokalemia: $a$ retrospective study in seven long-term care facilities. J Am Geriatr Soc, 1982. 30(5): p. 316-21.

34. Ko, B., et al., Sex differences in proximal and distal nephron function contribute to the mechanism of idiopathic hypercalcuria in calcium stone formers. Am J Physiol Regul Integr Comp Physiol, 2015. 309(1): p. R85-92.

35. Harris, A.N., et al., Differences in acidosis-stimulated renal ammonia metabolism in the male and female kidney. Am J Physiol Renal Physiol, 2019. 317(4): p. F890-F905.

36. Reilly, R.F. and C.L. Huang, The mechanism of hypocalciuria with NaCl cotransporter inhibition. Nat Rev Nephrol, 2011. 7(11): p. 669-74.

37. Shin, Y. and C.P. Brangwynne, Liquid phase condensation in cell physiology and disease. Science, 2017. 357(6357). 
38. McDonough, A.A., et al., Considerations when quantitating protein abundance by immunoblot. Am J Physiol Cell Physiol, 2015. 308(6): p. C426-33.

39. Bostanjoglo, M., et al., 11Beta-hydroxysteroid dehydrogenase, mineralocorticoid receptor, and thiazide-sensitive $\mathrm{Na}-\mathrm{Cl}$ cotransporter expression by distal tubules. $\mathrm{J}$ Am Soc Nephrol, 1998. 9(8): p. 1347-58.

40. Chen, J.C., et al., WNK4 kinase is a physiological intracellular chloride sensor. Proc Natl Acad Sci U S A, 2019. 116(10): p. 4502-4507.

41. McCormick, J.A., et al., Overexpression of the sodium chloride cotransporter is not sufficient to cause familial hyperkalemic hypertension. Hypertension, 2011. 58(5): p. 88894.

42. Wade, J.B., et al., Differential regulation of ROMK (Kir1.1) in distal nephron segments by dietary potassium. Am J Physiol Renal Physiol, 2011. 300(6): p. F1385-93.

43. Ong, J., et al., Renal sensory nerves increase sympathetic nerve activity and blood pressure in 2-kidney 1-clip hypertensive mice. J Neurophysiol, 2019. 122(1): p. 358-367. 
Table 1: Effects of KS-WNK1 on whole blood and urine parameters in female mice on low K+ diet

\begin{tabular}{r|cc|cc}
\hline & \multicolumn{2}{|c|}{ Control } & \multicolumn{2}{c}{ Low K+ } \\
\hline Whole Blood & WT & KS-WNK1-KO & WT & KS-WNK1-KO \\
Na+, mmol/L & $145.2(0.6)$ & $144.0(0.9)$ & $144.5(0.3)$ & $146.5(0.5){ }^{* *}$ \\
$\mathrm{~K}+, \mathrm{mmol} / \mathrm{L}$ & $4.2(0.3)$ & $4.2(0.1)$ & $3.3(0.1)$ & $2.9(0.1)^{*}$ \\
$\mathrm{Cl}-, \mathrm{mmol} / \mathrm{L}$ & $111.2(0.9)$ & $111.7(0.4)$ & $113.5(0.7)$ & $113.9(0.7)$ \\
$\mathrm{HCO}, \mathrm{mmol} / \mathrm{L}$ & $24.3(0.9)$ & $23.2(0.7)$ & $22.8(0.5)$ & $23.2(0.4)$ \\
$\mathrm{Ca}+, \mathrm{mmol} / \mathrm{L}$ & $1.31(0.03)$ & $1.32(0.02)$ & $1.30(0.02)$ & $1.28(0.03)$ \\
End Weight, g & $28.7(1.4)$ & $26.1(1.4)$ & $26.1(1.2)$ & $26.1(1.0)$ \\
\hline Metabolic Cage $\mathrm{n}=$ & 5 & 5 & 9 & 9 \\
Gel food intake, ml/d & $7.5(1.2)$ & $6.7(1.1)$ & $8.9(0.9)$ & $8.2(0.7)$ \\
water intake, ml/d & $1.1(0.1)$ & $1.1(0.1)$ & $1.7(0.6)$ & $1.7(0.4)$ \\
UNa+ ${ }^{*} \mathrm{~V}, \mathrm{mmol} / \mathrm{d}$ & $0.44(0.07)$ & $0.39(0.06)$ & $0.43(0.09)$ & $0.31(0.02)$ \\
UK+ ${ }^{*} \mathrm{~V}, \mathrm{mmol} / \mathrm{d}$ & $0.45(0.08)$ & $0.41(0.07)$ & $0.016(0.002)$ & $0.015(0.002)$ \\
U Osms, mOsms/kg & $1480(53)$ & $1437(81)$ & $1086(47)$ & $922(41) *$ \\
Spot Urine pH & $6.0(0.1)$ & $5.8(0.1)$ & $6.6(0.1)$ & $7.0(0.2)$ \\
Urine Volume, ml/d & $2.5(0.5)$ & $2.5(0.6)$ & $3.3(0.5)$ & $3.0(0.4)$ \\
\hline
\end{tabular}

Values are means (SE). Values are means (SE): Unpaired t-test were used to determine significant differences between WT and KS-WNK1-KO on the same diet, ${ }^{*} \mathrm{P} \leq 0.05$ and ${ }^{* *} \mathrm{P} \leq 0.01$.

Table 2: Effects of KS-WNK1 on whole blood and urine parameters in male mice on low $\mathrm{K}+$ diet

\begin{tabular}{r|cc|cc}
\hline & \multicolumn{2}{|c|}{ Control } & \multicolumn{2}{c}{ Low K+ } \\
\hline Whole Blood & WT & KS-WNK1-KO & WT & KS-WNK1-KO \\
Na+, mmol/L & $142.2(0.7)$ & $144.3(0.7)$ & $144.9(0.8)$ & $146.6(0.9)$ \\
$\mathrm{K}+, \mathrm{mmol} / \mathrm{L}$ & $4.5(0.1)$ & $4.3(0.2)$ & $2.7(0.1)$ & $2.9(0.1)$ \\
$\mathrm{Cl}-\mathrm{mmol} / \mathrm{L}$ & $113.2(1.5)$ & $111.9(0.5)$ & $109.2(1.7)$ & $108.0(0.9)$ \\
$\mathrm{HCO}, \mathrm{mmol} / \mathrm{L}$ & $22.8(0.7)$ & $23.2(0.5)$ & $24.6(1.1)$ & $25.8(0.5)$ \\
$\mathrm{Ca}+, \mathrm{mmol} / \mathrm{L}$ & $1.28(0.01)$ & $1.31(0.02)$ & $1.18(0.02)$ & $1.28(0.02){ }^{* *}$ \\
End Weight, g & $31.0(2.3)$ & $29.2(1.4)$ & $33.3(1.3)$ & $29.2(1.0)$ \\
\hline Metabolic Cage & 6 & 4 & 8 & 8 \\
Gel food intake, ml/d & $13.1(0.7)$ & $12.7(0.6)$ & $11.3(0.7)$ & $11.0(0.6)$ \\
water intake, ml/d & $0.9(0.1)$ & $0.9(0.1)$ & $1.5(0.3)$ & $1.6(0.2)$ \\
UNa+ ${ }^{*} \mathrm{~V}, \mathrm{mmol} / \mathrm{d}$ & $0.42(0.05)$ & $0.35(0.05)$ & $0.73(0.10)$ & $0.63(0.09)$ \\
UK+ ${ }^{*} \mathrm{~V}, \mathrm{mmol} / \mathrm{d}$ & $0.90(0.08)$ & $0.82(0.09)$ & $0.018(0.009)$ & $0.016(0.005)$ \\
U Osms, mOsms/kg & $1221(29)$ & $1268(45)$ & $831(56)$ & $725(42)$ \\
Spot Urine pH & $5.8(0.1)$ & $5.7(0.1)$ & $7.3(0.1)$ & $7.2(0.2)$ \\
Urine Volume, ml/d & $3.3(0.2)$ & $3.9(0.5)$ & $4.5(0.6)$ & $4.6(0.4)$ \\
\hline Valus
\end{tabular}

Values are means (SE): Unpaired t-test were used to determine significant differences between WT and KSWNK1-KO on the same diet, ${ }^{*} \mathrm{P} \leq 0.05$ and ${ }^{* *} \mathrm{P} \leq 0.01$. 


\section{Figure Legends}

Figure 1. KS-WNK1 expands the dynamic range of NCC phosphorylation in response to changes in blood [K+].

Immunoblot (IB) analysis of kidney cortical extracts from female and male WT and KS-WNK1-KO mice, subjected to dietary potassium maneuvers +/- diuretic challenge for 10 days.

(A) IBs of total NCC (tNCC) and active phospho thr-53 NCC (pNCC), from female and male mice fed control versus low $\mathrm{K}+$ diet.

(B) tNCC and pNCC IBs from female $(F)$ and male (M) mice fed control versus high $\mathrm{K}+$ diet plus amiloride $(2 \mathrm{mg} / \mathrm{kg} /$ day $)$

(C-E) Quantification of tNCC and pNCC protein abundance in male and female WT and KSWNK1-KO mice subjected to potassium maneuvers. All protein quantification was calculated as fold change compared to WT mice on control diet within the same western blot image, whose average value was set to 1 . Significance was determined by two-way ANOVA with post-hoc Sidak's multiple comparison test $\left({ }^{*} p \leq 0.05,{ }^{* *} p \leq 0.01\right)$. (C) Blood [K+] was inversely related to tNCC abundance; however, there was no significant difference between WT and KS-WNK1-KO mice. (D) On low K+ diets pNCC abundance was lower in KS-WNK1-KO mice. In contrast, during $\mathrm{K}+$ loading with amiloride, KS-WNK1-KO mice exhibited a relative increase in pNCC. (E) pNCC/tNCC ratio. The pNCC signal in WT mice reflects combined changes in phosphorylation and total protein abundance, whereas in KS-WNK1-KO mice changes in pNCC signal purely reflect changes in total protein abundance, resulting in pNCC/tNCC ratios near 1 regardless of the potassium maneuver.

(F-H) pNCC and tNCC abundance in individual male and female KS-WNK1-KO mice vs controls, plotted as a function of measured blood $[\mathrm{K}+]$ at the time of sacrifice. Linear regression analysis revealed differences between WT and KS-WNK1-KO mice for $(G)$ pNCC $(p=0.0277)$, and $(H)$ pNCC / tNCC ratio $(p<0.001)$.

Figure 2. Impaired WNK-SPAK/OSR1 pathway activation in K+ restricted KS-WNK1 knockout mice.

(A) Schematic illustrating WNK-SPAK/OSR1 pathway localization and activation during control (normokalemic) and low $\mathrm{K}+$ (hypokalemic) dietary conditions. During hypokalemia, the WNKSPAK/OSR1 pathway is activated and partially localized in WNK bodies.

(B-C) IBs of Kidney cortex homogenates from (B) female and (C) male WT and KS-WNK1-KO mice subjected to either control or low $\mathrm{K}+$ diets for $10 \mathrm{~d}$.

(D) Graphical summary of the results. The dotted line depicts control values normalized to 1.0. (Results are shown as means $\pm \mathrm{SE} ; n=6$ mice per genotype per diet. Two-way ANOVA with Sidak's multiple comparisons test was applied comparing WTand KS-WNK1-KO, ${ }^{*} \leq 0.05,{ }^{* *} P \leq 0.01$ ).

Figure 3. K+-restricted wild type and KS-WNK1 knockout mice exhibit sex differences in WNK body expression.

(A) Immunofluorescence (IF) of kidney sections from WT or KS-WNK1-KO mice treated with low $\mathrm{K}+$ diet for $10 \mathrm{~d}$. DCTs were identified by NCC co-staining and morphology. WNK4, 
pSPAK/pOSR1, and WNK1 antibodies colocalized within puncta in WT mice, whereas puncta were nearly absent in KS-WNK1-KO mice. All bars $=15 \mu \mathrm{m}$

(B) WNK body formation in female WT and KS-WNK1-KO mice. Puncta were largely absent in KS-WNK1-KO mice, though apical staining was still present. Uncommonly, mislocalized puncta containing pSPAK/pOSR1 and WNK4 were observed (arrowheads). Bar $=15 \mu \mathrm{m}$

(C) Imaris was used to quantify WNK body number and size (middle) and distance to lumen (right), from raw confocal IF images of pSPAK/pOSR1 intracellular puncta in DCTs (left). Bar $=4 \mu \mathrm{m}$.

(D-F) Quantification of pSPAK/pOSR1(D) puncta per cell (20 tubules per condition), (E) puncta diameter ( 5 tubules per condition), and $(F)$ distance to apical lumen in female and male mice (5 tubules per condition); ${ }^{*} \mathrm{P} \leq 0.05,{ }^{* *} \mathrm{P} \leq 0.01,{ }^{* * *} \mathrm{P} \leq 0.001,{ }^{* * * *} \mathrm{P} \leq 0.0001$, Two-way ANOVA with Sidak's multiple comparison.

\section{Figure 4. Effect KS-WNK1 on whole blood electrolytes and urine osmolality in K+ restricted female and male mice.}

Male and female WT and KS-WNK1-KO mice were fed low $\mathrm{K}+$ diet for 10 days and whole blood electrolytes and urine were obtained. Results were analyzed as combined (right) and sexdisaggregated data (middle and left)

(A) KS-WNK1-KO mice had significantly increased blood [Na+] when males and females were analyzed in combination, and when females were analyzes separately. Male KS-WNK1-KO mice had a higher mean blood $[\mathrm{Na}+]$ than sex-matched control littermates, but did not reach significance.

(B) Urine osmolality in KS-WNK1-KO mice was significantly decreased in the combined male and female dataset, and in females only. Mean $\mathrm{U}_{\text {osm }}$ in KS-WNK1-KO males was lower than male WT but did not reach significance.

(C) KS-WNK1 deletion had no significant effect on whole blood [K+] in the combined male and female pool, or in the male pool. However, female KS-WNK1-KO mice did have a significant decrease in whole blood $[\mathrm{K}+]$ compared to sex-matched controls.

(D) KS-WNK1 deletion had no significant effect on whole blood $\left[\mathrm{Ca}^{2+}\right]$ in the combined male and female pool or in the female pool. However, male KS-WNK1-KO mice did have a significant increase in whole blood $\left[\mathrm{Ca}^{2+}\right]$.

Sample size-For combined data: blood WT $n=22$ mice, KS-WNK1-KO $n=35$; urine WT $n=15$, KS-WNK1-KO $n=18$. Females: blood WT $n=12$, KS-WNK1-KO $n=17$; urine WT $n=8$, KS-WNK1$\mathrm{KO} n=9$. Males: blood WT $n=10, K S-W N K 1-K O n=18$; urine WT $n=7, K S-W N K 1-K O n=8)$. Unpaired t test was used to determine significance $\left({ }^{*} P \leq 0.05,{ }^{* *} P \leq 0.01\right)$.

\section{Figure 5. Female KS-WNK1 knockout mice are normotensive but thiazide-insensitive.}

Female KS-WNK1-KO shown in light red $(n=6)$, WT littermates shown in light blue $(n=4)$. Mice were subjected to either low $\mathrm{K}+$ or control diets for $10 \mathrm{~d}$, followed by supplementation with $1 \%$ NS in drinking water for $3 \mathrm{~d}$.

(A) KS-WNK1 expression had no significant effect on mean arterial pressure (MAP) in K+ restricted or control diet mice. Saline supplementation was associated with an increase in MAP in $\mathrm{K}+$ restricted mice, but no effect of KS-WNK1 genotype was observed. Each data point represents either daytime or nighttime MAP collected over $6 \mathrm{~h}$. Genotype had no significant effect based on two-way ANOVA. 
(B) Thiazide diuretic challenge. Mice were fed low $\mathrm{K}+$ diet for 10 days and then given $1 \%$ saline in their drinking water for $72 \mathrm{~h}$. Daytime MAP was measured for $6 \mathrm{~h}$ windows $24 \mathrm{~h}$ before HCTZ injection, 1h after HCTZ injection (+HCTZ, 25mg/kg IP) and $24 \mathrm{~h}$ after HCTZ injection. WT mice had a significant decrease in MAP with HCTZ treatment, whereas KS-WNK1-KO mice had a blunted response to HCTZ. ${ }^{*} \mathrm{P} \leq 0.05$, two-way ANOVA with post-hoc Sidak's multiple comparisons test.

Figure 6. KS-WNK1 is a DCT-specific signal amplifier.

(A) Graph depicting effect of KS-WNK1 on potassium-dependent NCC regulation. Wild type littermates exhibit a steep linear inverse relationship between NCC phosphorylation (activity) and blood [K+]. KS-WNK1 knockout mice exhibit a flattened $\mathrm{K}+$ response, such that the dynamic range of NCC activity is contracted. While KS-WNK1 suppresses NCC phosphorylation during hyperkalemia, its physiologic effects are most impactful during hypokalemia, when it acts as an NCC activator.

(B) During hypokalemia caused by dietary K+ restriction, KS-WNK1 normally facilitates WNK body formation and NCC activation via the WNK-SPAK/OSR1 pathway. In K+-restricted KS-WNK1 knockout mice, WNK bodies are absent or mislocalized, resulting in low SPAK/OSR1 and NCC activity. The effects of KS-WNK1 deletion were strongest in females, suggesting that WNK bodies contribute to sex-specific differences in tubular $\mathrm{Na}+$ transport. 
bioRxiv preprint doi: https://doi.org/10.1101/2021.03.12.435046; this version posted March 12, 2021. The copyright holder for this preprint (which was not certified by peer review) is the author/funder, who has granted bioRxiv a license to display the preprint in perpetuity. It is made available under aCC-BY-NC-ND 4.0 International license.

\section{Figure 1}

A

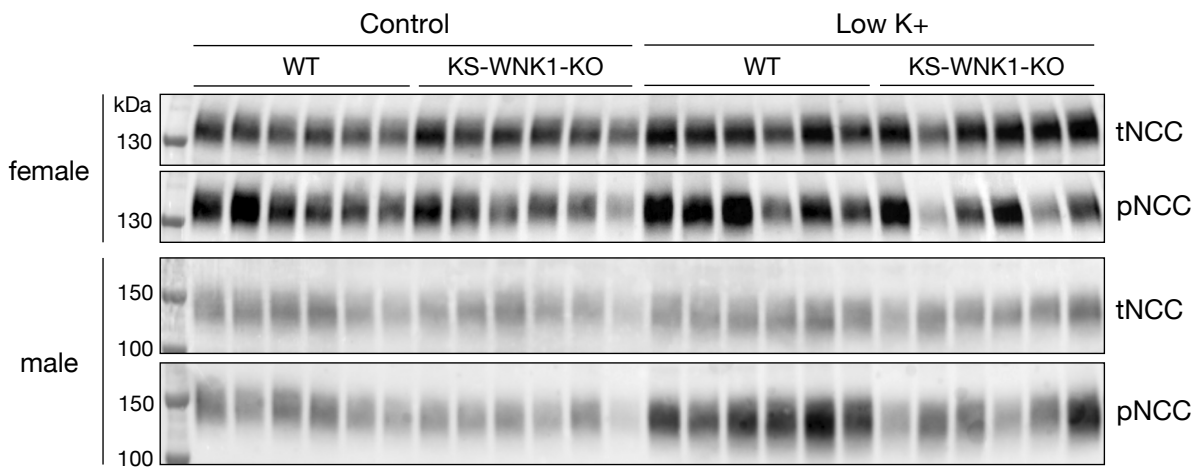

B

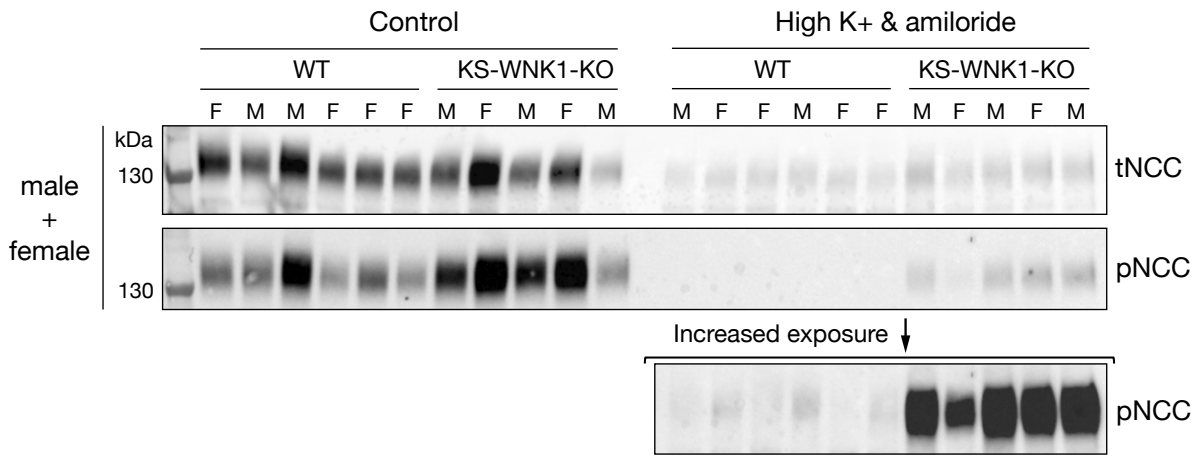

C

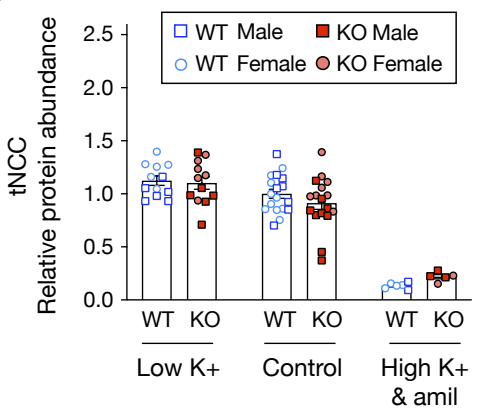

$\mathbf{F}$

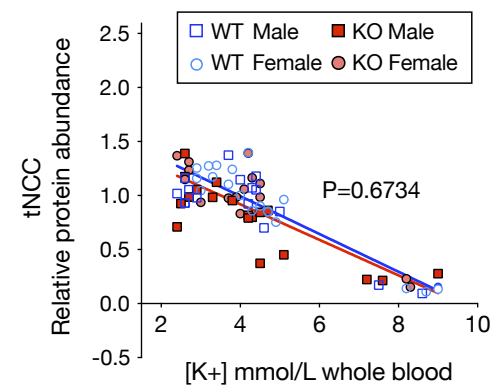

D

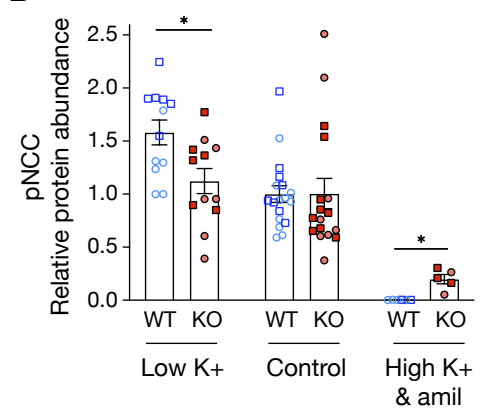

G

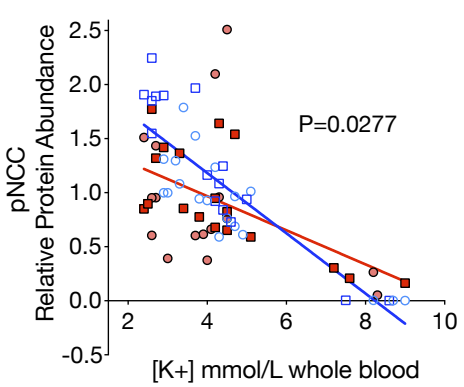

E

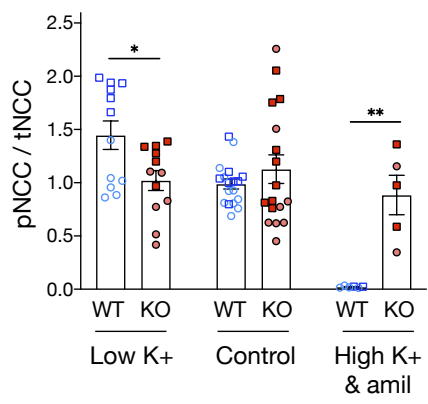

H

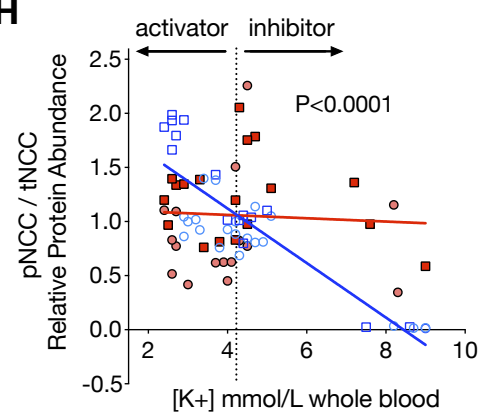


bioRxiv preprint doi: https://doi.org/10.1101/2021.03 12.435046; this version posted March 12, 2021. The copyright holder for this preprint (which was not certified by peer review) is the author/funder, who has granted bioRxiv a license to display the preprint in perpetuity. It is made available under aCC-BY-NC-ND 4.0 International license.

\section{Figure 2}

A

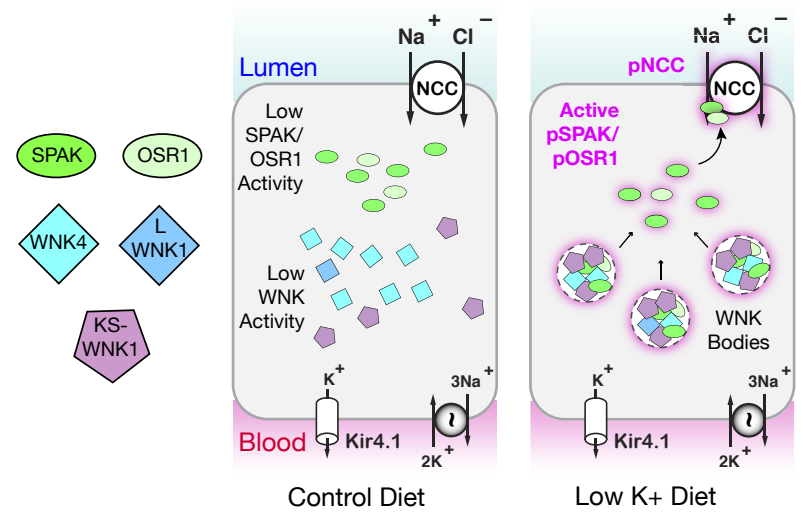

B

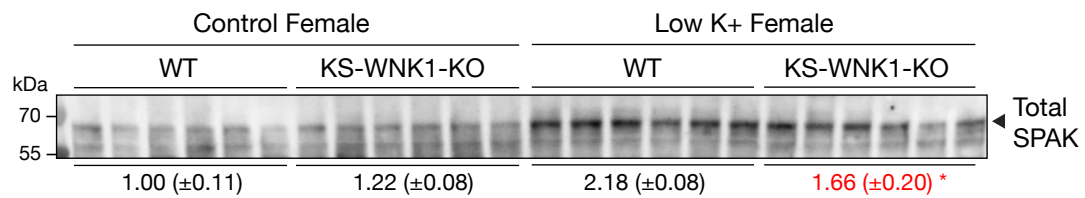

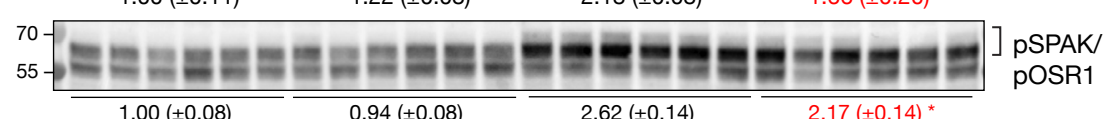

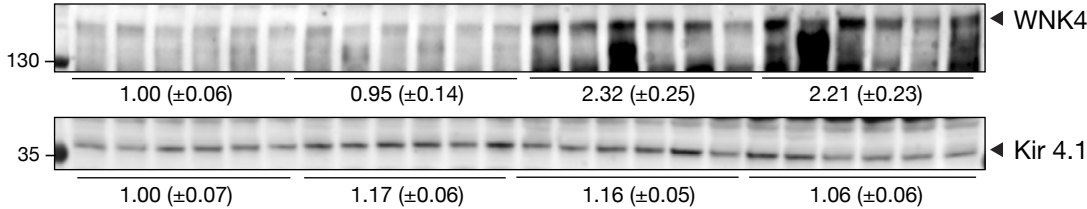

C
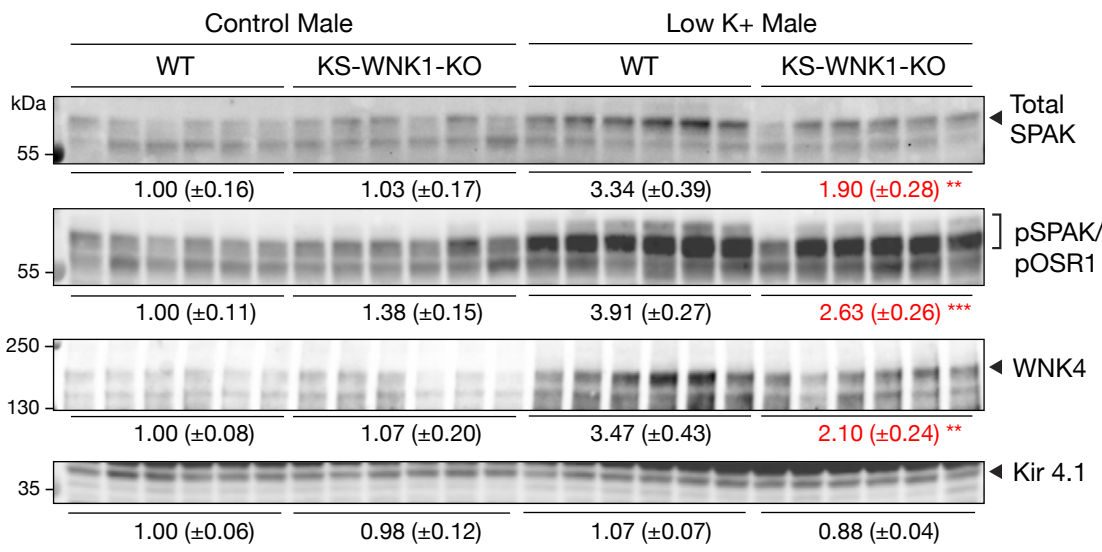

D

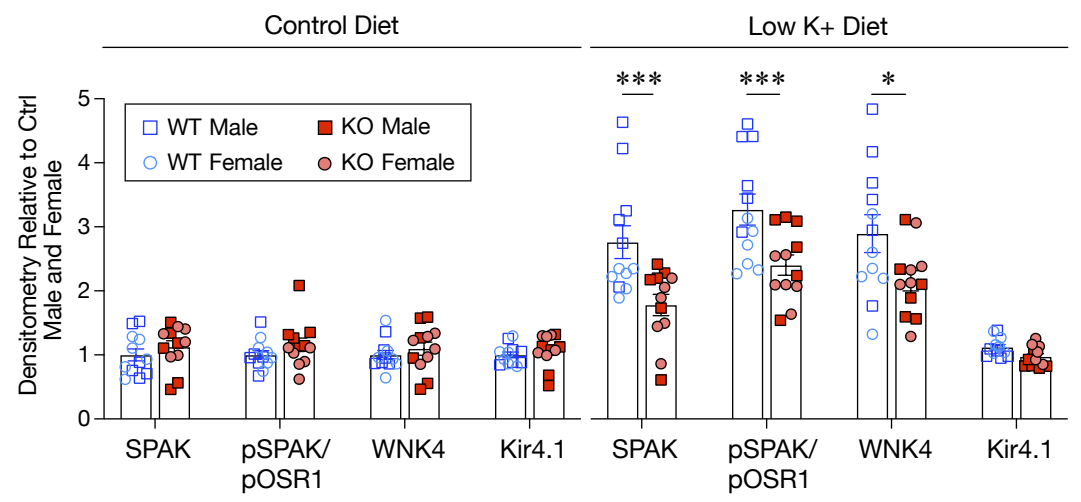


bioRxiv preprint doi: https://doi.org/10.1101/2021.03.12.435046; this version posted March 12, 2021. The copyright holder for this preprint (which was not certified by peer review) is the author/funder, who has granted bioRxiv a license to display the preprint in perpetuity. It is made available under aCC-BY-NC-ND 4.0 International license.

\section{Figure 3}

A

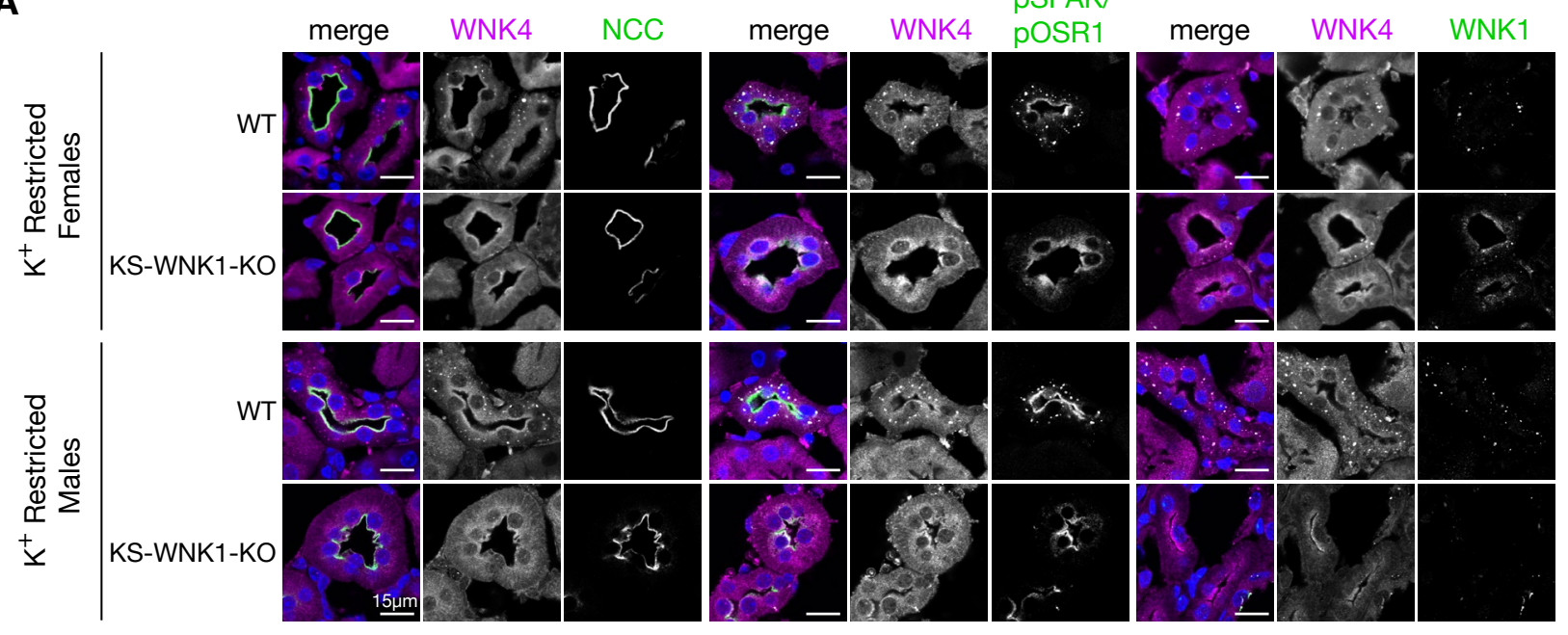

B

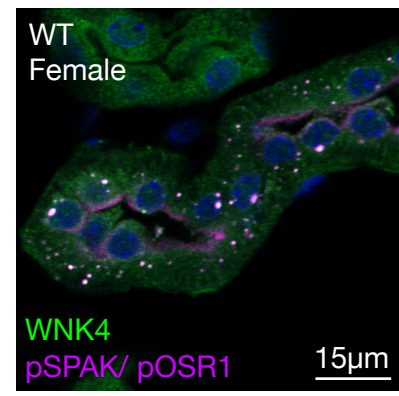

D

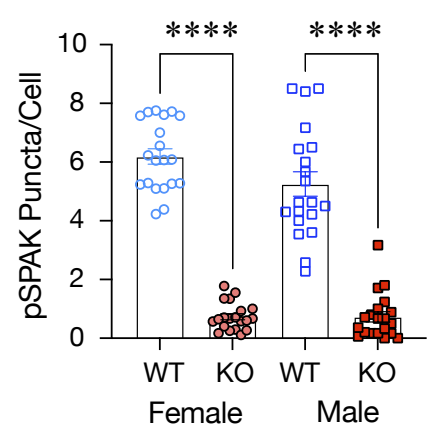

C

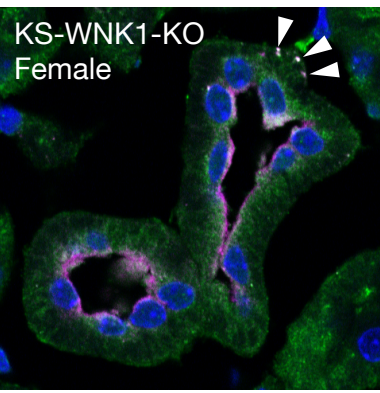

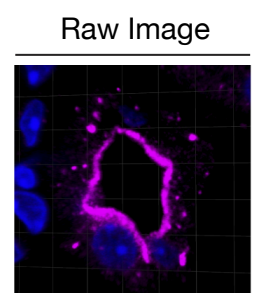

pSPAK/ pOSR1

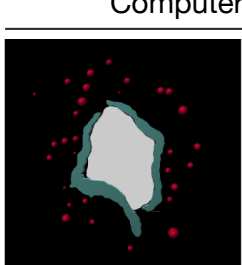

Puncta per Cell \& Diameter

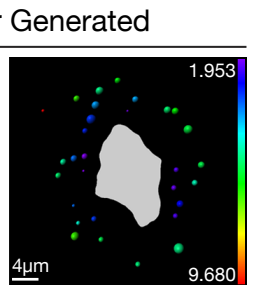

Distance to Lumen

F

E

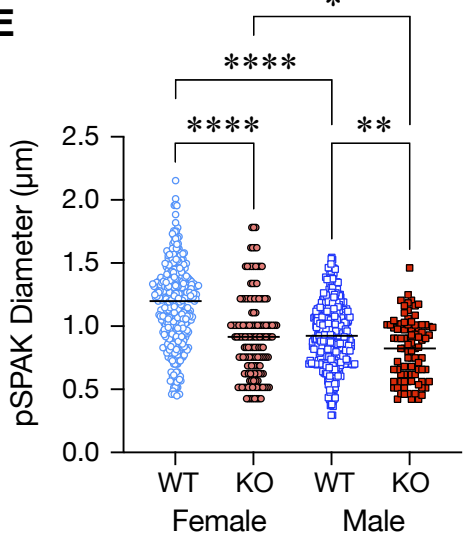

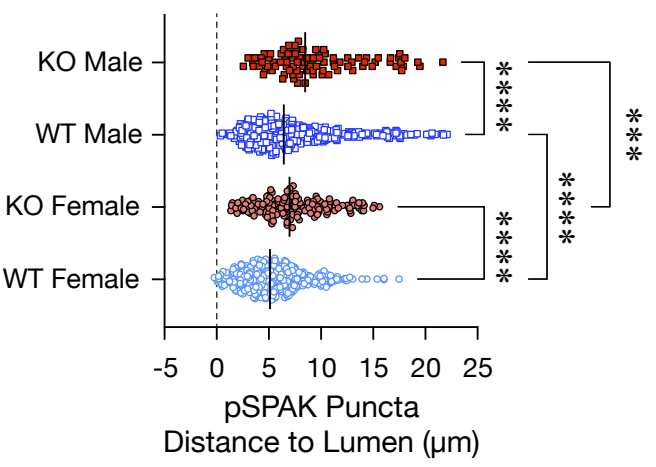


Figure 4

A

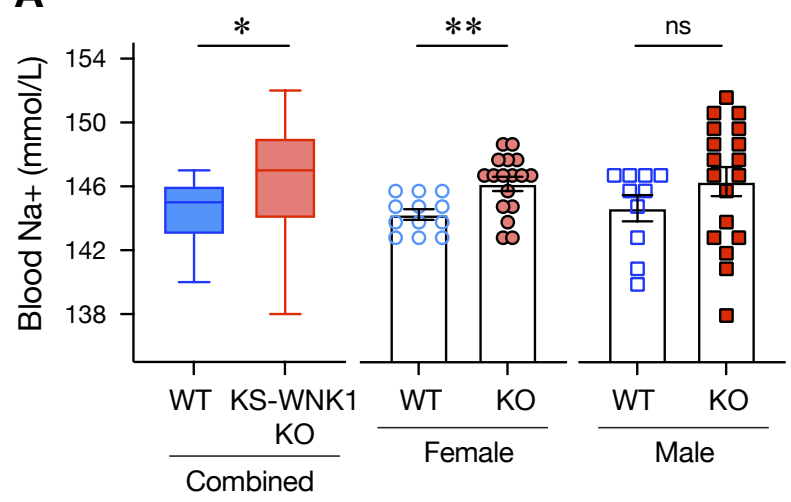

C

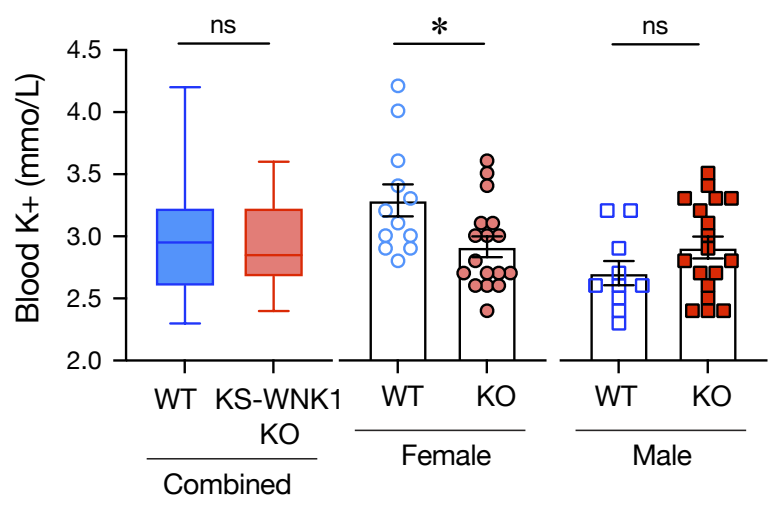

B

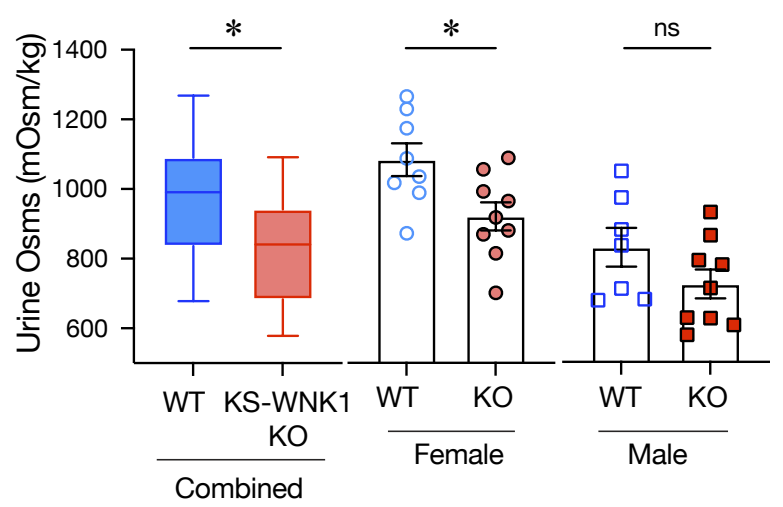

D

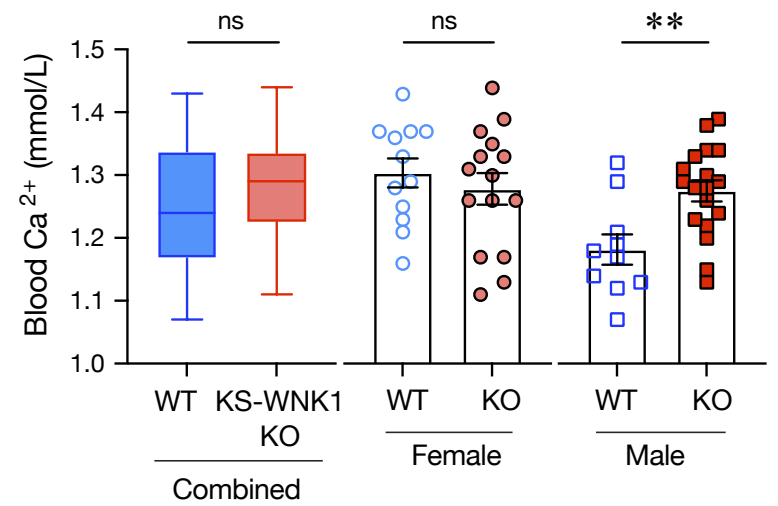




\section{Figure 5}

A

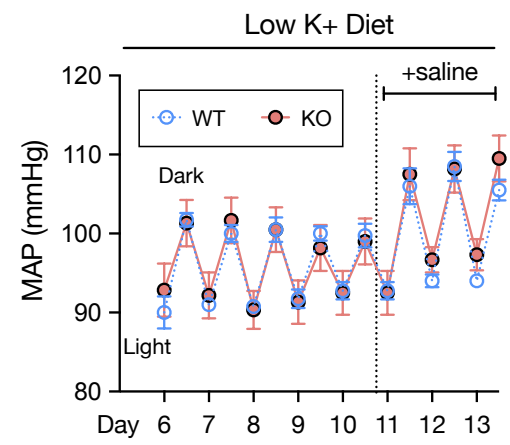

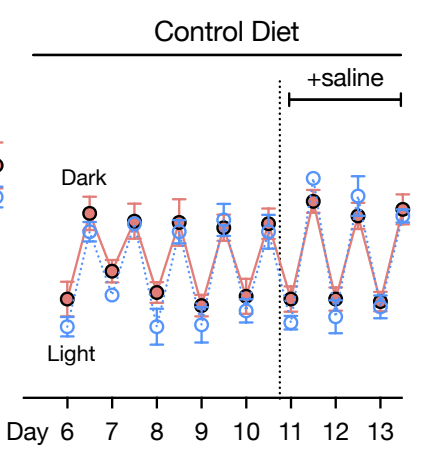

B

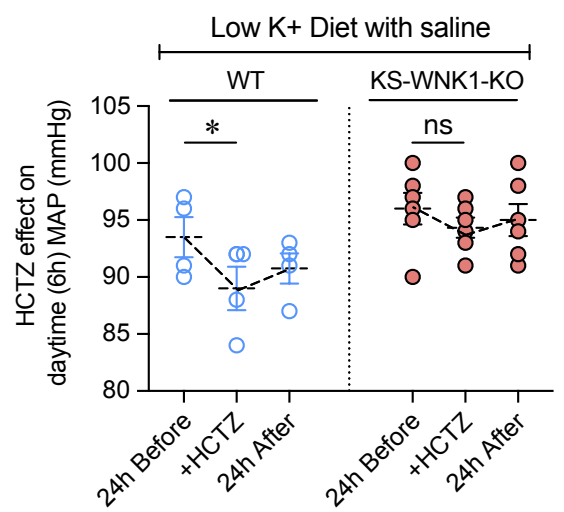

Figure 6

A

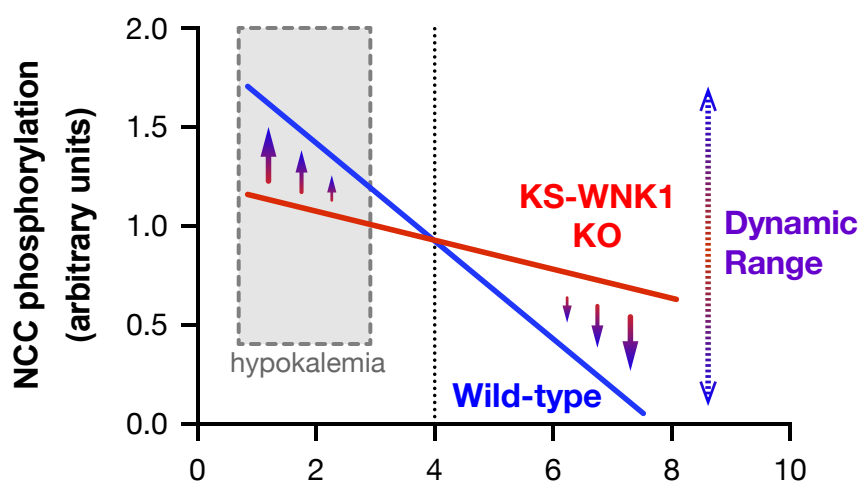

Plasma $[K+]$
B

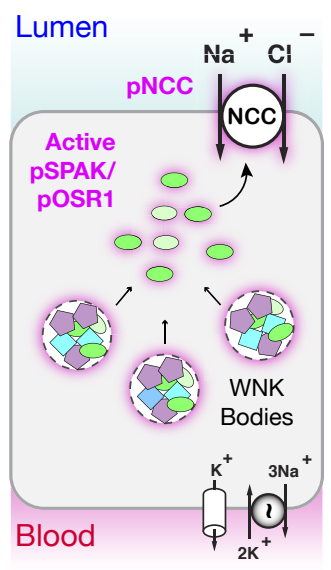

K+ restricted Wild-type

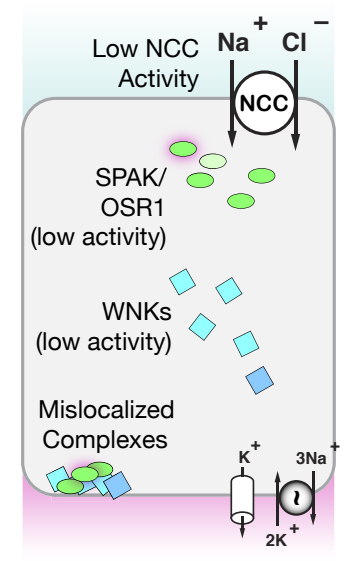

$\mathrm{K}+$ restricted KS-WNK1 KO 


\section{Supplemental Data}

Table S1: Effects of KS-WNK1 on whole blood and urine parameters in combined male and female mice on Low K+ diet

\begin{tabular}{r|cc|cc}
\hline & \multicolumn{2}{|c|}{ Control } & \multicolumn{2}{c}{ Low K+ } \\
& WT & KS-WNK1-KO & WT & KS-WNK1-KO \\
\hline Whole Blood $\mathrm{n}=$ & 12 & 20 & 22 & 35 \\
$\mathrm{Na}+, \mathrm{mmol} / \mathrm{L}$ & $143.7(0.6)$ & $144.2(0.6)$ & $144.7(0.4)$ & $146.5(0.5){ }^{*}$ \\
$\mathrm{~K}+, \mathrm{mmol} / \mathrm{L}$ & $4.3(0.1)$ & $4.2(0.1)$ & $3.0(0.1)$ & $2.9(0.1)$ \\
$\mathrm{Cl}-\mathrm{mmol} / \mathrm{L}$ & $112.2(0.9)$ & $111.8(0.3)$ & $111.5(1.0)$ & $110.4(0.7)$ \\
$\mathrm{HCO}, \mathrm{mmol} / \mathrm{L}$ & $23.6(0.6)$ & $23.2(0.4)$ & $23.6(0.6)$ & $24.5(0.4)$ \\
$\mathrm{Ca}+, \mathrm{mmol} / \mathrm{L}$ & $1.30(0.02)$ & $1.32(0.02)$ & $1.25(0.02)$ & $1.28(0.01)$ \\
End Weight, g & $29.8(1.3)$ & $27.7(1.0)$ & $29.3(1.2)$ & $27.7(0.8)$ \\
\hline Metabolic Cage $\mathrm{n}=$ & 11 & 9 & 17 & 17 \\
Gel food intake, ml/d & $10.5(1.1)$ & $9.3(1.2)$ & $10.1(0.6)$ & $9.5(0.6)$ \\
water intake, ml/d & $1.0(0.1)$ & $1.0(0.1)$ & $1.4(0.2)$ & $1.6(0.2)$ \\
UNa+ ${ }^{*} \mathrm{~V}, \mathrm{mmol} / \mathrm{d}$ & $0.43(0.04)$ & $0.37(0.04)$ & $0.56(0.07)$ & $0.47(0.06)$ \\
UK+ ${ }^{*} \mathrm{~V}, \mathrm{mmol} / \mathrm{d}$ & $0.70(0.09)$ & $0.59(0.09)$ & $0.017(0.004)$ & $0.015(0.003)$ \\
U Osms, mOsms/kg & $1350(48)$ & $1369(57)$ & $967(49)$ & $824(37){ }^{*}$ \\
Spot Urine pH & $5.9(0.09)$ & $5.8(0.08)$ & $7.0(0.10)$ & $7.1(0.11)$ \\
Urine Volume, ml/d & $2.9(0.3)$ & $3.1(0.5)$ & $3.8(0.4)$ & $3.8(0.3)$ \\
\hline
\end{tabular}

Values are means (SE). Values are means (SE): Unpaired t-test were used to determine significant differences between WT and KS-WNK1 KO on the same diet, * $\mathrm{P} \leq 0.05$ and ${ }^{* *} \mathrm{P} \leq 0.01$. 
bioRxiv preprint doi: $\mathrm{https}$ //doi.org/10.1101/2021.03.12.435046; this version posted March 12, 2021. The copyright holder for this preprint (which was not certified by peer review) is the author/funder, who has granted bioRxiv a license to display the preprint in perpetuity. It is made available under aCC-BY-NC-ND 4.0 International license.

\section{Control vs Low K+ Diet: Females}

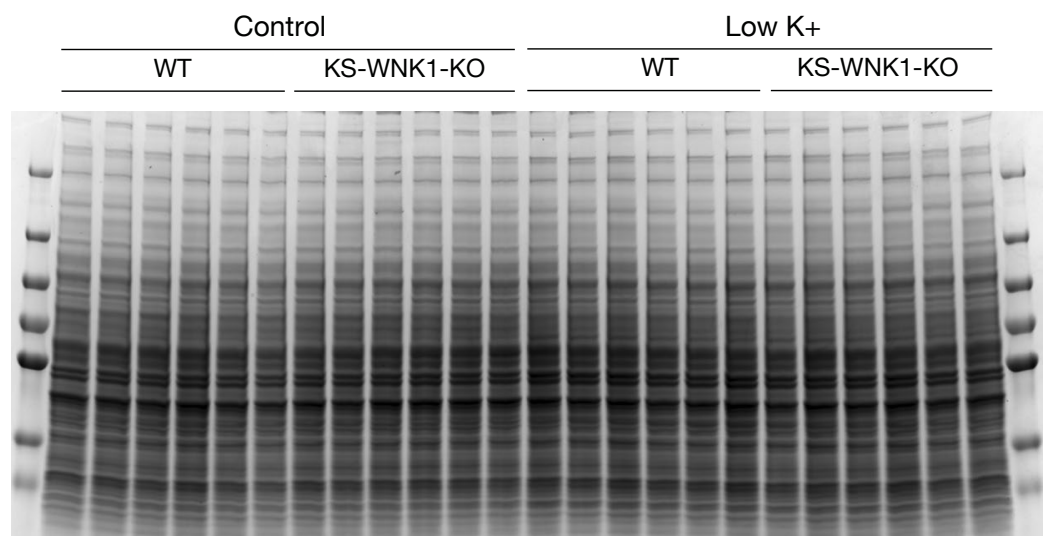

Control vs Low K+ Diet: Males

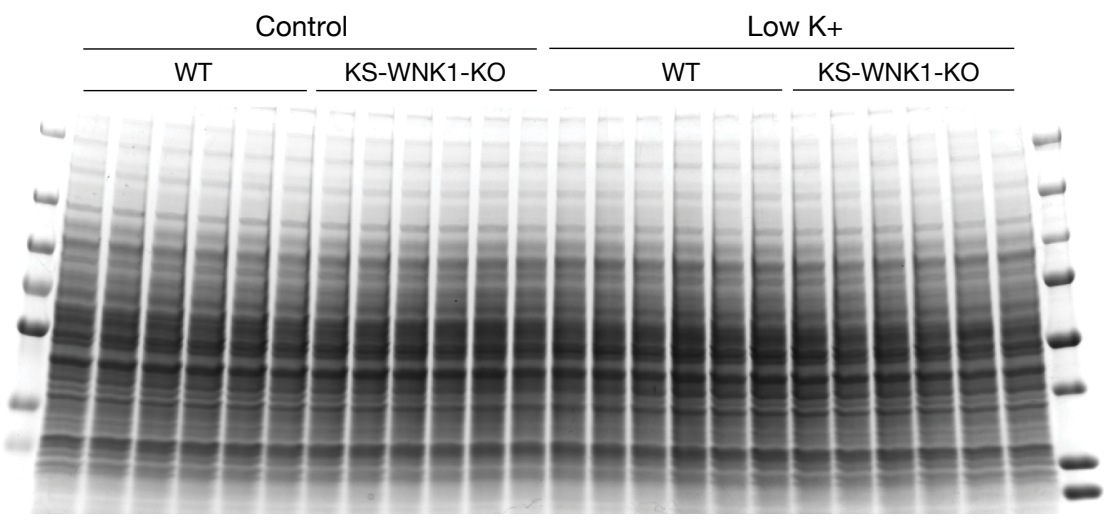

Control vs High K+ / Amiloride Treatment: Males + Females

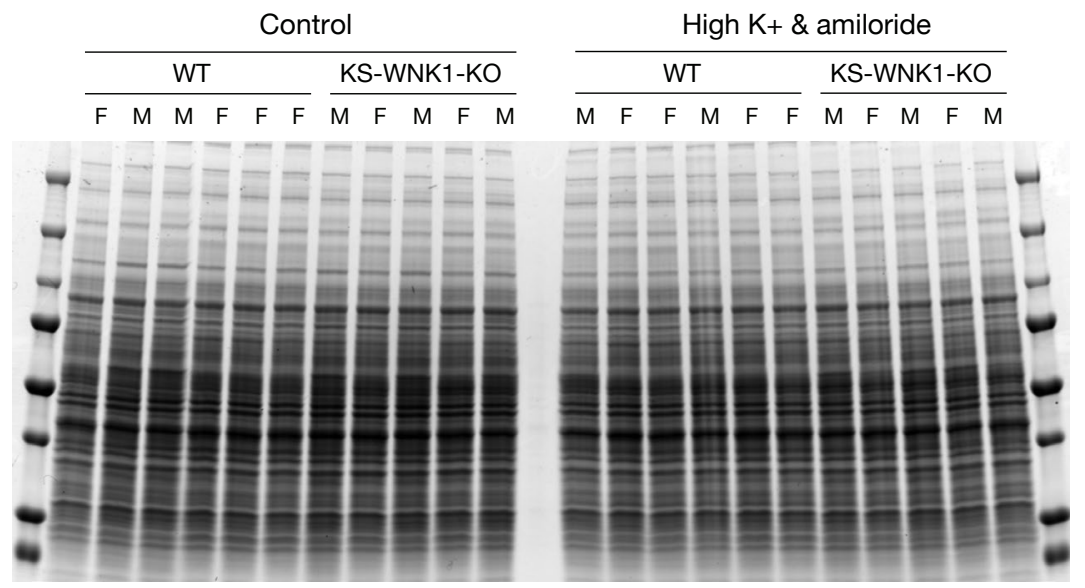

Figure S1. Optimized Coomassie Gels for Western Blotting.

Gels correspond to data presented in Figures 1 and 2. 


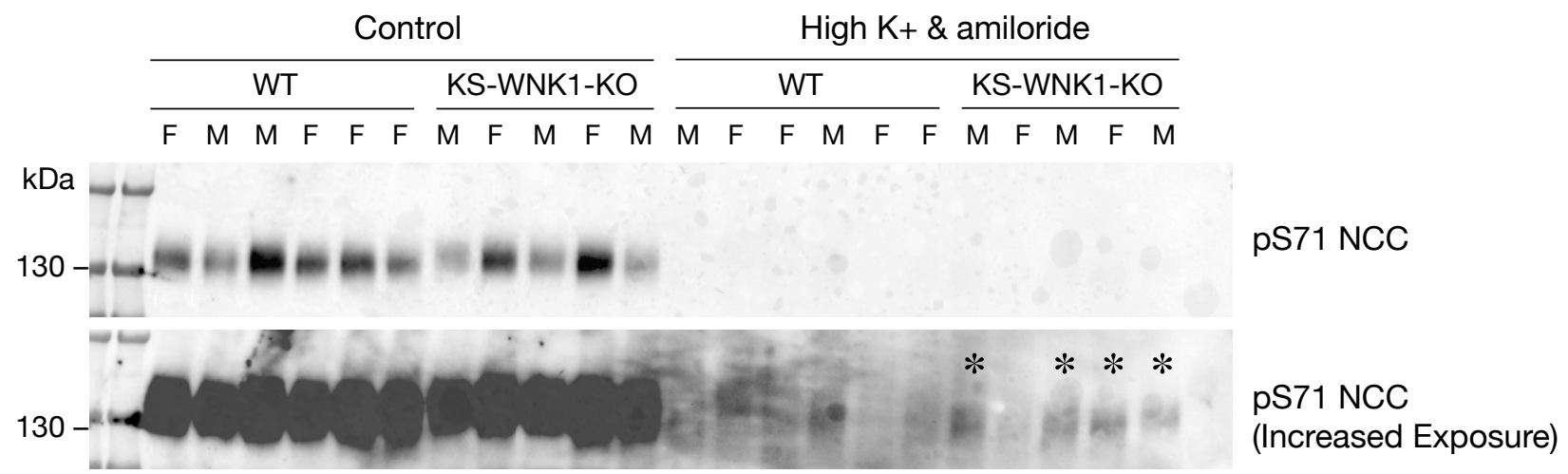

Figure S2. pSer71 antibody detects increased pNCC in KS-WNK1-KO mice subjected to potassium loading with amiloride.

Immunodetectable pNCC bands in KO mice under K+ loaded conditions are indicated with asterisks. 
A
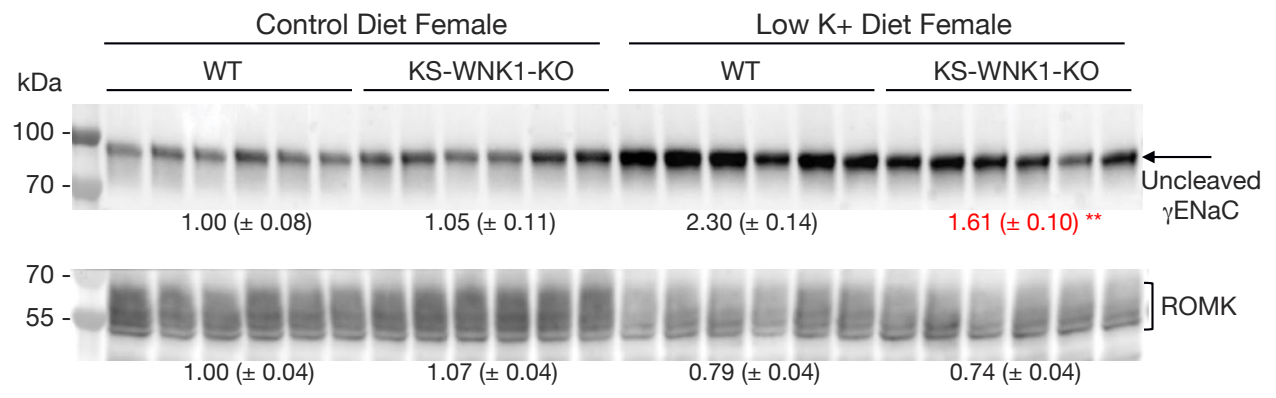

B

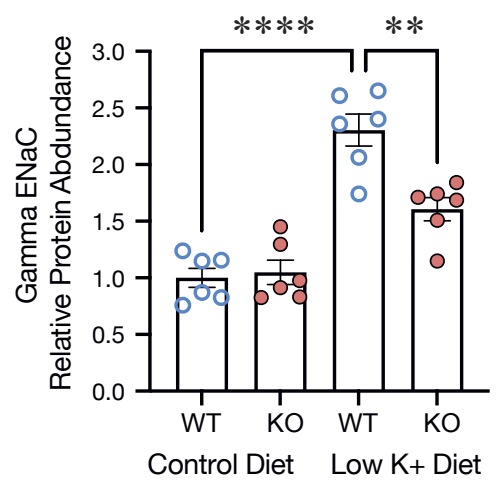

C

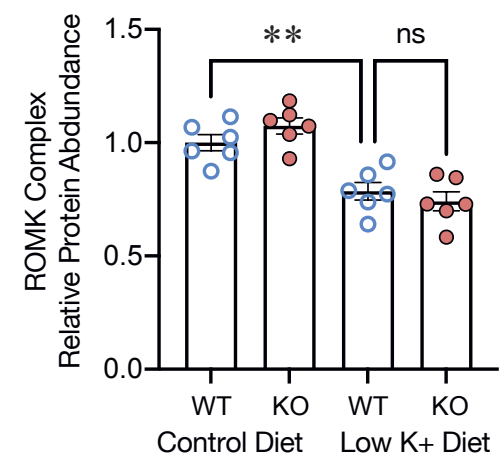

D

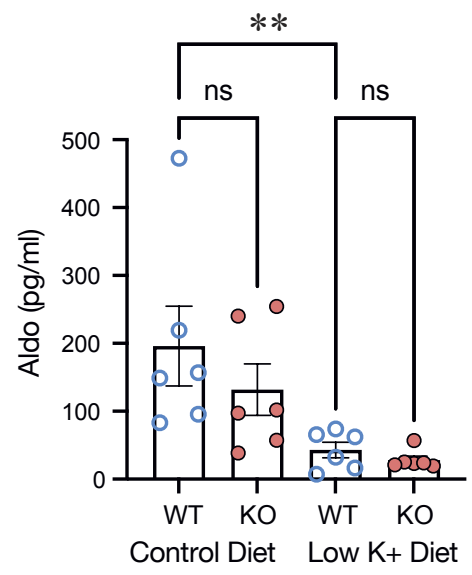

Figure S3. Effects of KS-WNK1 deletion on ENaC, ROMK, and aldosterone levels in female mice.

(A) Immunoblots of gENaC and ROMK in kidney cortical homogenates of female WT and KSWNK1-KO mice subjected to control or low $\mathrm{K}+$ diets for $10 \mathrm{~d}$.

(B) Quantification of gENaC protein abundance.

(C) Quantification of ROMK protein abundance

(D) Quantification of plasma aldosterone concentrations.

${ }^{* *} \mathrm{P} \leq 0.01,{ }^{* * *} \mathrm{P}<0.001$, ns= not significant; all comparisons by Two-way ANOVA with Sidak's post-hoc test for multiple comparisons. 